\title{
Identification and characterization of a 25- IncRNA prognostic signature for early recurrence in hepatocellular carcinoma
}

Yi Fu ${ }^{1,2,3}$, Xindong Wei ${ }^{4}$, Qiuqin Han ${ }^{1,2}$, Jiamei Le ${ }^{1,2}$, Yujie Ma ${ }^{2}$, Xinjie Lin ${ }^{2}$, Yuhui X ${ }^{5}$, Ning Liu ${ }^{6}$, Xuan Wang ${ }^{7}$, Xiaoni $\mathrm{Kong}^{8}$, Jinyang $\mathrm{Gu}^{9^{*}}$, Ying Tong ${ }^{10^{*}}$ and Hailong $\mathrm{Wu}^{1,2^{*}}$

\begin{abstract}
Background: Early recurrence is the major cause of poor prognosis in hepatocellular carcinoma (HCC). Long noncoding RNAs (IncRNAs) are deeply involved in HCC prognosis. In this study, we aimed to establish a prognostic IncRNA signature for HCC early recurrence.

Methods: The IncRNA expression profile and corresponding clinical data were retrieved from total 299 HCC patients in TCGA database. LncRNA candidates correlated to early recurrence were selected by differentially expressed gene (DEG), univariate Cox regression and least absolute shrinkage and selection operator (LASSO) regression analyses. A 25-IncRNA prognostic signature was constructed according to receiver operating characteristic curve (ROC). Kaplan-Meier and multivariate Cox regression analyses were used to evaluate the performance of this signature. ROC and nomogram were used to evaluate the integrated models based on this signature with other independent clinical risk factors. Gene set enrichment analysis (GSEA) was used to reveal enriched gene sets in the high-risk group. Tumor infiltrating lymphocytes (TILs) levels were analyzed with single sample Gene Set Enrichment Analysis (ssGSEA). Immune therapy response prediction was performed with TIDE and SubMap. Chemotherapeutic response prediction was conducted by using Genomics of Drug Sensitivity in Cancer (GDSC) pharmacogenomics database.
\end{abstract}

\footnotetext{
* Correspondence: gjynyd@126.com; lilytongy@hotmail.com; wuhl@sumhs.edu.cn

${ }^{9}$ Department of Transplantation, Xinhua Hospital Affiliated to Shanghai Jiao Tong University School of Medicine, Shanghai 200092, China

${ }^{10}$ Department of Liver Surgery, Renji Hospital Affiliated to Shanghai Jiao Tong University School of Medicine, Shanghai 200127, China

${ }^{1}$ Affiliated Zhoupu Hospital, Shanghai University of Medicine and Health Sciences, Shanghai 201318, China

Full list of author information is available at the end of the article
}

(c) The Author(s). 2021 Open Access This article is licensed under a Creative Commons Attribution 4.0 International License, which permits use, sharing, adaptation, distribution and reproduction in any medium or format, as long as you give appropriate credit to the original author(s) and the source, provide a link to the Creative Commons licence, and indicate if changes were made. The images or other third party material in this article are included in the article's Creative Commons licence, unless indicated otherwise in a credit line to the material. If material is not included in the article's Creative Commons licence and your intended use is not permitted by statutory regulation or exceeds the permitted use, you will need to obtain permission directly from the copyright holder. To view a copy of this licence, visit http://creativecommons.org/licenses/by/4.0/ The Creative Commons Public Domain Dedication waiver (http://creativecommons.org/publicdomain/zero/1.0/) applies to the data made available in this article, unless otherwise stated in a credit line to the data. 
Results: Compared to low-risk group, patients in high-risk group showed reduced disease-free survival (DFS) in the training $(p<0.0001)$ and validation cohort $(p=0.0132)$. The 25 -IncRNA signature, AFP, TNM and vascular invasion could serve as independent risk factors for HCC early recurrence. Among them, the 25-IncRNA signature had the best predictive performance, and combination of those four risk factors further improves the prognostic potential. Moreover, GSEA showed significant enrichment of "E2F TARGETS", "G2M CHECKPOINT", "MYC TARGETS V1" and "DNA REPAIR" pathways in the high-risk group. In addition, increased TILs were observed in the low-risk group compared to the high-risk group. The 25-IncRNA signature negatively associates with the levels of some types of antitumor immune cells. Immunotherapies and chemotherapies prediction revealed differential responses to PD-1 inhibitor and several chemotherapeutic drugs in the low- and high-risk group.

Conclusions: Our study proposed a 25-IncRNA prognostic signature for predicting HCC early recurrence, which may guide postoperative treatment and recurrence surveillance in HCC patients.

Keywords: Long non-coding RNA signature, Hepatocellular carcinoma, Early recurrence, Tumor infiltrating lymphocytes

\section{Background}

The very recent epidemiologic study has shown that liver cancer ranks the sixth commonly diagnosed cancer and the fourth leading cause of cancer death in the world. An estimated 84,100 liver cancer cases occurred and 78,200 liver cancer cases died in 2018 [1]. Hepatocellular carcinoma (HCC) compromises $75-85 \%$ of primary liver cancer [1]. The main clinical curative treatments for HCC include liver transplantation, percutaneous radiofrequency ablation and liver resection, among which liver resection is the most employed treatment [2]. Although 5-year overall survival rate reaches up to $50 \%$, recurrence occurs in more than $70 \%$ HCC patients after curative surgery [3]. Clinically, the recurrence within 2-year after resection is defined as early recurrence, whereas the recurrence $>2$-year is defined as late recurrence. Compared to late recurrence, HCC patients with early recurrence usually showed poorer prognosis [4].

Currently, many approaches, such as the TNM staging system of the American Joint Committee on Cancer (AJCC), the Barcelona Clinic Liver Cancer (BCLC) classification, and the Cancer of the Liver Italian Program (CLIP) staging system, have been employed to evaluate the prognosis of HCC patients [5]. However, their assessment criteria mainly rely on the clinicopathological features of HCC patients but do not take into account the critical and complicated molecular pathogenesis, an important factor in determining the outcome of HCC. Therefore, their prognostic predictive performance was unsatisfactory [6]. Meanwhile, serum alpha-fetoprotein (AFP) detection and medical imaging techniques are clinically used for postsurgery surveillance of recurrence in HCC patients, but with limited effectiveness due to the low specificity and sensitivity of those surveillance means [7].

The advent of high throughput array/sequencing and high-efficiency big data analysis in past decades makes it possible and reliable to construct multi-gene signatures to evaluate prognosis and predict therapeutic response in cancer patients. For example, a 70-gene signature had been established to aid decision making of adjuvant chemotherapy in patients with estrogen receptorpositive early breast cancer $[8,9]$. More importantly, this 70-gene-signature based diagnostic test known as "MammaPrint" (Agendia, Amsterdam, The Netherlands) has been approved by the Food and Drug Administration (FDA) to predict breast cancer recurrence [10], and been validated in several retrospective studies [11, 12]. Additionally, an 18-gene signature ColoPrint (Agendia, Amsterdam, The Netherlands) was developed to predict disease relapse in patients with early-stage colorectal cancer (CRC) [13], and had been validated in other independent studies $[14,15]$. Several multi-gene signatures have been constructed in HCC for prognosis evaluation. For example, Wei et al. developed a 20-miRNA signature to predict post-surgery survival in HCC patients [16]; Nault et al. constructed a 5-gene signature to evaluate the overall survival in HCC patients [17]; Kim et al. established a 233-gene signature to predict late recurrence in HCC patients [18]. Recently, prognostic signatures based on specific groups of genes such as glycolyis-related genes, metabolic-related genes and autophagy-related genes were also reported [19-21]. However, those multi-gene signatures of HCC mainly focus on overall survival and later recurrence, and few multi-gene signatures have been established to predict early recurrence in HCCs.

Long non-coding RNAs (lncRNAs) are a class of transcripts that are longer than 200 nucleotides (nt) and do not encode proteins [22]. Accumulating evidence has indicated the involvement of lncRNAs in diverse biological processes and disease pathogenesis [23]. Moreover, some IncRNAs have been reported to contribute to the initiation and progression in HCCs. For example, lncRNA- 
ANRIL has been reported to promote hepatocarcinoma cell proliferation [24]; and IncRNA-MALAT1 could function as a proto-oncogene to transform hepatocytes and enhance hepatocarcinoma cell growth [25]. In addition, some lncRNAs have been demonstrated to associate with HCC prognosis. For example, the overexpression of lncRNA-MVIH was associated with poor recurrence-free survival and overall survival in HCC patients [26]; LncRNA-PTTG3P expression was positively associated with tumor size, TNM stage and poor survival in HCC patients [27]. Although lncRNAs are involved in the progression and associated with prognosis in HCCs, lncRNA-based gene signatures for HCC prognostic evaluation, especially for early recurrence, are limited.

In this study, we analyzed the expression profile of lncRNAs and their association with early recurrence in the Liver Hepatocellular Carcinoma (LIHC) project from The Cancer Genome Atlas (TCGA) database (TCGALIHC). We constructed a 25-lncRNA signature significantly associated with $\mathrm{HCC}$ early recurrence. Based on this multi-lncRNA signature, HCC patients can be classified into low- and high-risk groups according to their risk scores. The early recurrence rate was significantly higher in the high-risk group than in the low-risk group. Moreover, the risk score negatively correlated with recurrence-free survival in HCC patients. Multivariate Cox regression analysis demonstrated that the 25lncRNA signature, serum AFP, TNM stage and vascular invasion were 4 independent risk factors of HCC early recurrence. Compared with the other 3 risk factors, the 25-IncRNA signature had the best predictive performance for HCC early recurrence. Furthermore, the 25lncRNA signature could synergize with serum AFP, TNM stage and vascular invasion to improve the prognosis evaluation for HCC early recurrence. In addition, in the context of this 25-lncRNA risk signature, we demonstrated that the "E2F TARGETS", "G2M CHECKPOINT", "MYC TARGETS V1" and "DNA REPAIR" were the most significantly enriched gene sets in the high-risk group. Moreover, the low-risk group showed greater tumor-infiltrating lymphocytes (TILs) compared to the high-risk group, and the 25-IncRNA prognostic signature was significantly negatively associated with the potent antitumor immune cells (i.e. type $1 \mathrm{~T}$ helper cell, effector memory CD8 $\mathrm{T}$ cell and activated CD8 $\mathrm{T}$ cell). Finally, the low-risk group was predicted to be more sensitive to immunotherapy like anti-PD-1 and chemotherapies like docetaxel, gefitinib and vinblastine, while the high-risk group was predicted to be more sensitive to doxorubicin, mitomycin $\mathrm{C}$ and paclitaxel. In conclusion, our findings may provide some insight into lncRNA-based personalized treatment and improve the strategy of post-surgery recurrence surveillance in HCC patients.

\section{Methods}

\section{TCGA-LIHC database preparation and IncRNA profile} mining

Gene expression profile of $\mathrm{HCC}$ and corresponding clinical information were downloaded from TCGA-LIHC (http://cancergenome.nih.gov/). Total 314 out of all 374 HCC samples with complete follow-up information (overall survival (OS) time, OS status, disease free survival (DFS) time and status) were retained. Among these 314 patients, some patients' follow-up time was less than 1 month, and their OS and DFS status were labeled as "alive" and "recurrence free". Therefore, these patients were not suitable for early recurrence analysis and they were excluded. Thus, we used 299 patients for signature construction in this study. The $299 \mathrm{HCC}$ patients were then randomly divided into a training cohort $(N=150)$ and a validation cohort $(N=149)$. Based on the information of annotated lncRNAs in GENCODE V30, 14,847 human lncRNAs with Ensembl gene ID were obtained and their corresponding expression profile was extracted from the TCGA-LIHC.

\section{Construction and validation of IncRNA-based risk signature}

Most bioinformatics analyses were conducted using $R$ software. DEG analysis was performed between the 150 HCC samples in the training cohort and 50 normal tissue samples from TCGA-LIHC project by using $\mathrm{R}$ package "edgeR" $[28,29]$. Univariate Cox regression analysis was performed to select early recurrence related lncRNAs by using R package "survival" [30]. FunRich (version 3) was used to draw Venn diagram between differentially expressed lncRNAs and early recurrence related lncRNAs to obtain candidate lncRNAs for signature construction [31]. Candidate lncRNAs were then further analyzed in LASSO regression analysis by running R package "glmnet" for 1000 times [32], and the most powerful prognostic lncRNAs were selected through 10-fold cross-validation with lambda.min as the optimized cut-off [33]. Risk score of each patient was calculated in a linear combination of lncRNAs weighted by their corresponding regression coefficients and expression levels in indicated HCC patients by formula (riskscore $=\sum$ coefficient $\times$ expression $($ gene $)$ ). Receiver operating characteristic curve (ROC) analysis was conducted by using $\mathrm{R}$ package "pROC" [34], and the predictive performance was assessed by calculating the area under curve (AUC). Finally, a combination of 25 lncRNAs was chosen for establishing risk signature because this 25-lcnRNA risk signature gave the largest AUC in ROC analysis. The $150 \mathrm{HCC}$ patients were divided into the low-risk group $(N=75)$ and the high-risk group $(N=75)$ by using the median risk score as cut-off. A correlation analysis was performed between the risk 
score and early recurrence. Kaplan-Meier analysis, cumulative hazard and cumulative events analyses were conducted by using R package "survival" in the training cohort, the validation cohort and the total 299 HCC patients to investigate the early recurrence survival between low risk patients and high risk patients. Univariate and multivariate Cox analysis were done in the total 299 HCC patients with $\mathrm{R}$ package "survival" to evaluate whether the risk score could serve as an independent factor for early recurrence prediction in HCCs. Nomogram was constructed by using the 25-lncRNA signature, AFP, vascular invasion, TNM stages and their corresponding multivariate Cox regression coefficients, and calibration plots were generated with $\mathrm{R}$ package "regplot" [35]. C-index was used to evaluate the model performance for predicting early recurrence.

\section{Gene set enrichment analysis (GSEA)}

GSEA was conducted by using GSEA JAVA program (version 4.0.3) downloaded from official website (http:// software.broadinstitute.org/gsea/index.jsp) to find out enriched gene sets. MsigDB h.all.v7.1.symbols.gmt gene set collection was chosen for identifying hallmarks of HCC early recurrence. The random sample permutations were set to be 1000 with the significance set as $|\mathrm{NES}|>1$, FDR $\mathrm{q}<0.25$ and nominal $P<0.05$.

\section{Analysis of the levels of tumor-infiltrating lymphocytes and immune therapy response prediction}

Immune infiltration analysis was performed with single sample Gene Set Enrichment Analysis (ssGSEA) by using "GSVA" package in R [36]. A group of 28 cellmarker sets were used for calculating normalized enrichment score (NES) for each cell type in every 299 HCC samples [37]. Correlation analysis between risk scores and NES of immune cells was performed by function "cor.test" in R. TIDE (Tumor Immune Dysfunction and Exclusion) algorithm and SubMap modules from GenePattern were used to predict the response to immune checkpoint blockade for all 299 HCC samples [38-40].

\section{Analysis of chemotherapeutic response prediction}

Chemotherapeutic response prediction for every 299 HCC samples was conducted in R by using "pRRophetic" package based on the Genomics of Drug Sensitivity in Cancer (GDSC) pharmacogenomics database. The half maximal inhibitory concentration $\left(\mathrm{IC}_{50}\right)$ was estimated by ridge regression and the prediction accuracy was evaluated by 10 -fold cross-validation [41].

\section{Real time quantitative RT-PCR}

To validate the 25-IncRNA signature in clinical samples, 3 lncRNAs from the signature were selected and their relative expressions in HCC samples were detected by
RT-qPCR. Total RNA from 36 paired HCC tumor and adjacent tissues provided by Xinhua Hospital were extracted by using TRIzol (Invitrogen, 15596026) according to the manufacturer's instructions. CDNA was synthesized by using ReverTra Ace $^{\circ}$ qPCR RT Master Mix with gDNA Remover (TOYOBO, FSQ-301) in a SimpliAmp Thermal Cycler (Applied Biosystems). The $20 \mu \mathrm{L}$ PCR reaction system consist of $2 \mu \mathrm{L}$ cDNA, $0.8 \mu \mathrm{L}$ forward primer, $0.8 \mu \mathrm{L}$ reverse primer, $10 \mu \mathrm{L}$ CYBR Premix Ex TaqII, 0.4 $\mu \mathrm{L}$ ROX Reference Dye II and $6 \mu \mathrm{L}$ deionized water (Takara CYBR Premix Ex TaqII, RR820A). RT-PCR was performed in ABI Biosystems ${ }^{\text {Tm }} 7500$ RealTime qPCR System (Applied Biosystems). 18s was used as a housekeeping gene for normalization and the relative expression of selected genes was calculated by using $2^{-\Delta \Delta C T}$ method. Primers used were synthesized by GENEWIZ and the sequences of primers were ENSG00000231918 (GTGGCTCTGCCTTGGGTAAT, TTCCAGAACAACCTTGTCAGA), ENSG00000248596 (GCCAGAATTGGCGGTTTCTC, ATCGCTGAGTGT GTCGAGTG), and ENSG00000223392 (ATCCTTAC CCTGCATTGCCC, ATGATCCAACCATCTGCAGG $\mathrm{G).}$

\section{Statistical analysis}

DeLong's test was used to compare the sensitivity and specificity of two ROC curves. Chi-square test was used to evaluate the impact of risk score group distribution on recurrence cases between 1 year and 2 years. The correlation of risk scores with disease free survival (DFS), NES of tumor-infiltrating lymphocytes and levels of immune checkpoints was analyzed by nonparametric Spearman's rank correlation analysis. The log-rank test was used for Kaplan-Meier survival analyses, cumulative hazard and cumulative events analyses. The Cox proportional hazards regression model was used for univariate and multivariate analyses. Wilcoxon test was used for comparing NES of immune cells and $\mathrm{IC}_{50}$ of drugs between the low-risk and high-risk group. The difference was considered statistically significant when $P<0.05$ in all statistical analysis.

\section{Results}

HCC dataset preparation and identification of candidate IncRNAs from the training cohort

HCC RNA-seq data and corresponding clinical information were downloaded from the TCGA-LIHC (Liver Hepatocellular Carcinoma) project. After removal of samples without complete survival information, total 299 out of all 374 HCC samples were enrolled in this study for further analysis. Table S1 shows the clinical characteristics of the $299 \mathrm{HCC}$ samples, in which more than $50 \%$ HCC patients had recurrence. Because there are no suitable GEO datasets which are comparable to 
the TCGA-LIHC project containing comprehensive data on both lncRNA expression profile and patients' clinical characteristics, we then randomly divided the 299 HCC patients into a training cohort $(n=150)$ and a validation cohort $(n=149)$ by using "split" function in R software instead of setting an external validation cohort. Bioinformatics analyses were first performed in the training cohort and further validated in the validation cohort (Fig. 1A).

To establish a lncRNA-based risk signature, differentially expressed gene (DEG) analysis on lncRNAs was performed between the training cohort and 50 normal controls from the TCGA-LIHC project. A total of 1495 DEG IncRNAs were found significantly dysregulated in HCC samples (1159 up-regulated and 336 downregulated, $\log 2|\mathrm{FC}|>1, \quad \mathrm{FDR}<0.05$ ) (Fig. $1 \mathrm{~B}$ and $\mathrm{C}$ ). Meanwhile, a univariate Cox regression analysis revealed that total 1973 lncRNAs were associated with HCC early recurrence (ER lncRNAs) $(P<0.05)$ (Fig. 1D). Finally, a Venn diagram between the 1495 DEG IncRNAs and the 1973 ER lncRNAs identified 358 lncRNA candidates which may have potential prognostic value for HCC early recurrence (Fig. 1D).

\section{Pilot construction of multi-IncRNA signatures for HCC early recurrence}

The least absolute shrinkage and selection operator (LASSO) Logistic Regression is a selection and shrinkage technique designed for regression model initially applied to Ordinary Logistic Regression [42]. LASSO can better identify those risk factors strongly linked to the outcome and is widely employed in signature construction [43]. To identify key lncRNAs suitable for establishing a risk signature for predicting HCC early recurrence, those 358 candidate lncRNAs (Fig. 1D) were further analyzed in LASSO regression. A total of 1000 LASSO regression iterations were performed by using the $R$ package "glmnet". Lambda.min was chosen as the optimized cutoff to select key lncRNAs for risk model (Fig. S1) [44]. Consequently, 7 lncRNA combinations were obtained after LASSO analysis (Table S2 and Fig. S1). Thus, 7 lncRNA risk signatures were individually constructed based on these combinations. The risk score of each HCC patient was calculated in a linear formula risk score $=\sum$ coefficient $\times$ expression $($ gene $) \quad($ expression: lncRNA expression in individual HCC patients; coefficient: regression coefficients of indicated lncRNAs). To determine which lncRNA risk signature gives the best predictive performance on early recurrence, receiver operating characteristics (ROC) analysis was conducted between the 7 lncRNA risk signatures. As shown in Fig. $1 \mathrm{E}$, all the 7 lncRNA risk signatures gave high area under the ROC curve (AUC, AUC > 80\%), suggesting the reliability of our LASSO analysis. Among them, the 25-
lncRNA risk signature gave the highest AUC (AUC = $86.70 \%$ ) (Fig. 1F), suggesting the 25-lncRNA risk signature has the best predictive performance for $\mathrm{HCC}$ early recurrence.

\section{Risk score calculation of the 25-IncRNA risk signature}

Since the 25-lncRNA risk signature gave the best predictive performance, we then selected this signature to establish a risk model for HCC early recurrence. The detailed information of the 25 lncRNAs, including Ensembl gene ID, gene symbol, hazard ratio and coefficients, was summarized in Table 1. Among them, 19 lncRNAs (ENSG00000253417, ENSG00000272205, ENSG00000269894, ENSG00000275437, ENSG00000223392, ENSG00000248596, ENSG00000268201, ENSG00000247675, ENSG00000231918, ENSG00000234129, ENSG00000269974, ENSG00000236366, ENSG00000275223, ENSG00000253406, ENSG00000232079, ENSG00000255980, ENSG00000267905, ENSG00000176912, ENSG00000254333) had positive coefficients and were negatively associated with disease free survival (DFS), and the remainder 6 lncRNAs (ENSG00000259834, ENSG00000254887, ENSG00000259974, ENSG00000273837, ENSG00000231246, ENSG00000234283) had negative coefficients and were positively associated with DFS (Table 1). Here, we named those lncRNAs with positive coefficients as risk lncRNAs and those with negative coefficients as protective lncRNAs. The risk score could be calculated according to the coefficients of individual lncRNAs and their expression in corresponding HCC patients.

\section{The 25-IncRNA risk signature correlates with HCC early recurrence}

To determine whether the 25-lncRNA risk signature could predict $\mathrm{HCC}$ early recurrence, we first calculated the risk scores of the $150 \mathrm{HCC}$ patients in the training cohort and then distributed them according to their risk scores from low to high (Fig. 2A). The median risk score was set as the cut-off to separate those patients into low-risk group ( $n=75$, patients' risk scores $<$ the median risk score) and high-risk group ( $n=75$, patients' risk scores $>$ the median risk score) (Fig. 2A). As shown in Fig. 2B, the 19 risk lncRNAs were mostly enriched in the high-risk group whereas the 6 protective lncRNAs were mainly enriched in the low-risk group. Moreover, $81.25 \%$ of recurrence cases in 1 -year and $76.06 \%$ in 2 year came from the high-risk group, while the percentages were respectively 18.75 and $23.94 \%$ in the low-risk group (Fig. 2C). These results indicate that the 25lncRNA risk signature have satisfying predictive potential for HCC early recurrence in the training cohort.

\section{Validation of the 25-IncRNA risk signature}

To validate the predictive potential of the 25-lncRNA risk signature, we evaluated it in the validation cohort. According to the median cut-off in the training cohort, the 

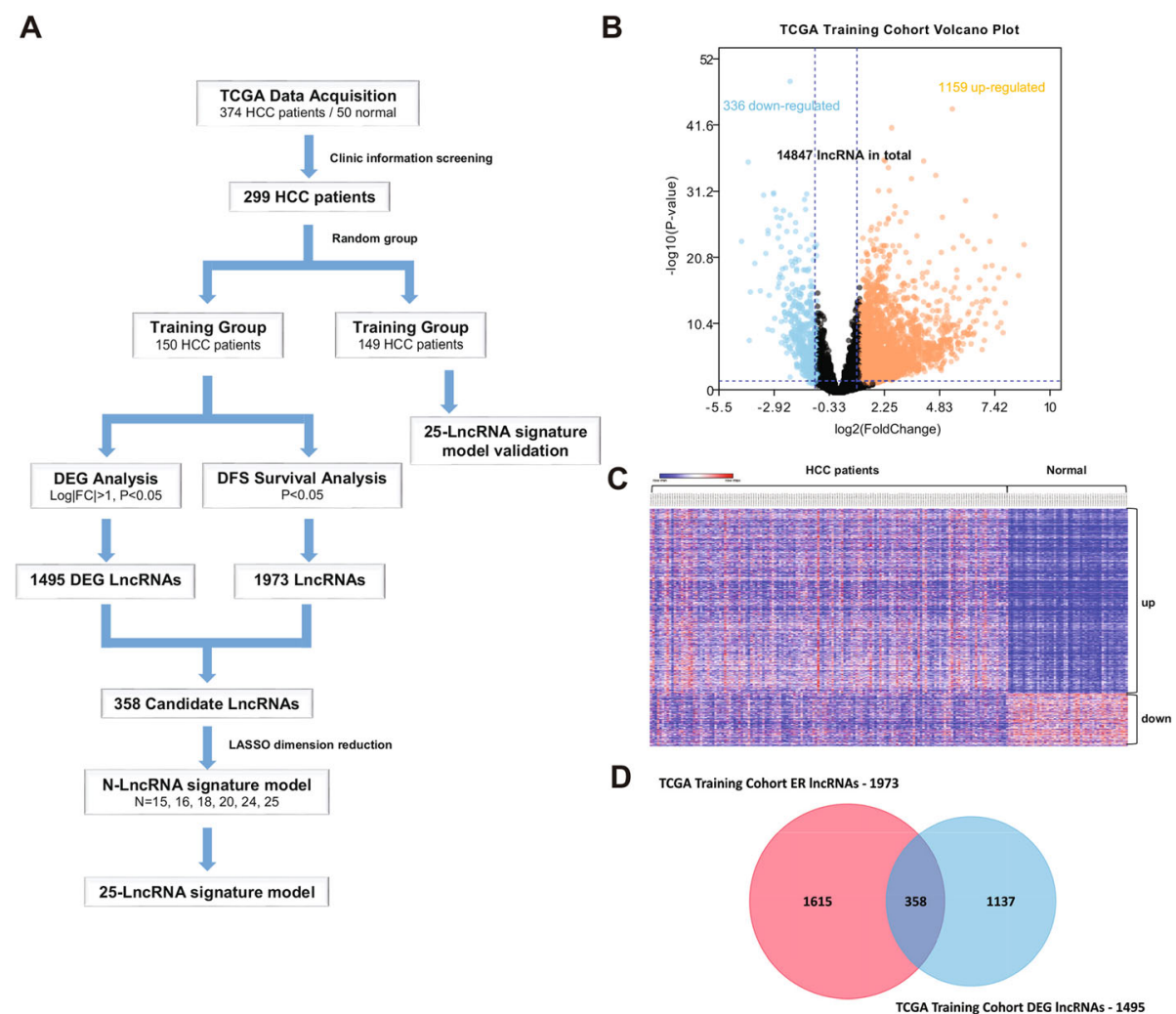

E

$\mathbf{F}$
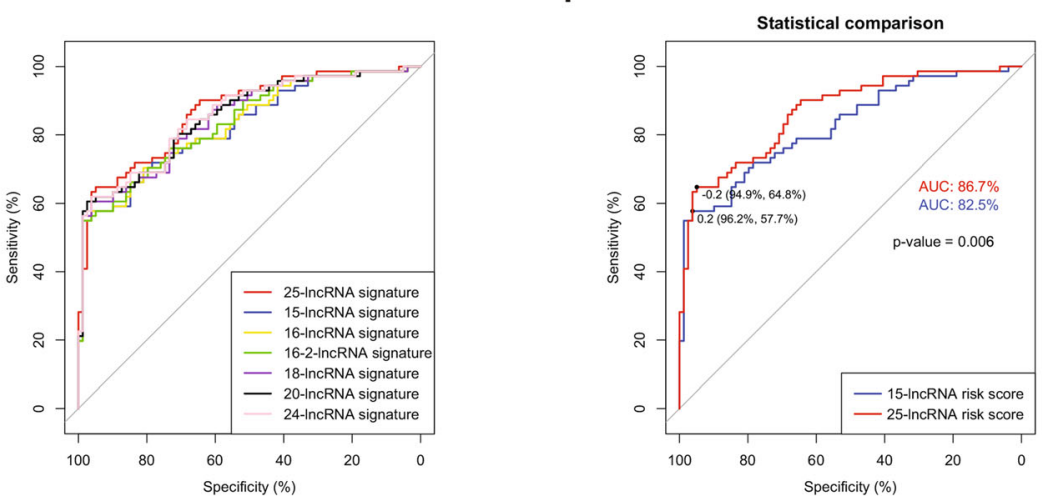

Fig. 1 Data processing and IncRNA-based early risk signature construction from candidate IncRNAs. A) Schematic diagram of data processing and construction of IncRNA-based signature; B) Volcano plot of IncRNAs expression in the TCGA training cohort. Differentially expressed gene (DEG) analysis shows 1159 up-regulated IncRNAs and 336 down-regulated IncRNAs; C) Heatmap of 1495 DEG IncRNAs in 150 HCC samples and 50 normal tissues; D) Venn plot of DEG IncRNAs and early recurrence related IncRNAs (ER IncRNAs) in the TCGA training cohort. 358 DEG IncRNAs with potential prognostic value for HCC early recurrence were identified; E) ROC plot of 7 IncRNA signatures; F) ROC plot comparison between the 15 -IncRNA risk signature and the 25-IncRNA risk signature, $P=0.006$

validation cohort $(n=149)$ was separated into the low-risk group $(n=69)$ and the high-risk group $(n=80)$ (Fig. 3A). In line with the finding in the training cohort, the risk lncRNAs were mainly enriched in the high-risk group whereas the protective ones were mainly enriched in the low-risk group (Fig. 3B). Meanwhile, $69.09 \%$ of 1-year recurrence cases and $63.01 \%$ of 2-year recurrence cases came from the high-risk group (Fig. 3C). Moreover, the predictive potential of the 25-lncRNA risk signature was also evaluated in the total 299 recruited HCC patients. Similarly, 299 HCC patients were separated into low-risk group $(n=$ $144)$ and high-risk group $(n=155)$ according to the median cut-off in the training cohort (Fig. 3D). Most of the risk lncRNAs were enriched in the high-risk group and most of the protective lncRNAs were enriched in the low-risk group (Fig. 3E). Consistent with this finding, non-pair Wilcoxon test confirmed enrichment of the risk lncRNAs and the protective lncRNAs in the high-risk and low-risk groups 
Table 1 LncRNAs significantly associated with the disease free survival in the training cohort patients $(N=150)$

\begin{tabular}{|c|c|c|c|c|c|c|c|}
\hline Ensembl & Gene Symbol & $\begin{array}{l}P \\
\text { value } \\
\mathrm{a}\end{array}$ & $\begin{array}{l}\text { Hazard } \\
\text { Ratio }^{a}\end{array}$ & Coefficient & associated diseases & Description & Reference \\
\hline ENSG00000253417 & LINC02159 & $\begin{array}{l}< \\
0.001\end{array}$ & 2.85 & 0.069 & $\begin{array}{l}\text { colorectal cancer, melanoma, head and neck squamous cell } \\
\text { carcinoma }\end{array}$ & $\begin{array}{l}\text { long intergenic } \\
\text { non-protein cod- } \\
\text { ing RNA } 2159\end{array}$ & {$[1-3]$} \\
\hline ENSG00000272205 & & $\begin{array}{l}<.001 \\
0.00\end{array}$ & 2.47 & 0.233 & NR & NR & \\
\hline ENSG00000269894 & & $\begin{array}{l}< \\
0.001\end{array}$ & 2.48 & 0.092 & NR & NR & \\
\hline ENSG00000275437 & & $\begin{array}{l}< \\
0.001\end{array}$ & 4.78 & 0.083 & NR & NR & \\
\hline ENSG00000223392 & CLDN10-AS1 & $\begin{array}{l}< \\
0.001\end{array}$ & 1.82 & 0.278 & $\begin{array}{l}\text { atherogenesis, lung adenocarcinoma, colorectal cancer, thyroid } \\
\text { cancer, cholangiocarcinoma, colon adenocarcinoma }\end{array}$ & $\begin{array}{l}\text { CLDN10 antisense } \\
\text { RNA } 1\end{array}$ & {$[4-6,20]$} \\
\hline ENSG00000248596 & LOC643201 & $\begin{array}{l}< \\
0.001\end{array}$ & 1.91 & 0.335 & colorectal cancer & $\begin{array}{l}\text { centrosomal } \\
\text { protein } 192 \mathrm{kDa} \\
\text { pseudogene }\end{array}$ & [5] \\
\hline ENSG00000268201 & & $\begin{array}{l}< \\
0.001\end{array}$ & 1.98 & 0.330 & NR & NR & \\
\hline ENSG00000247675 & LRP4-AS1 & $\begin{array}{l}< \\
0.001\end{array}$ & 7.36 & 0.567 & breast cancer, pancreatic neuroendocrine tumour & $\begin{array}{l}\text { LRP4 antisense } \\
\text { RNA } 1\end{array}$ & [7] \\
\hline ENSG00000231918 & LOC730100 & $\begin{array}{l}< \\
0.001\end{array}$ & 2.19 & 0.339 & glioma & $\begin{array}{l}\text { uncharacterized } \\
\text { LOC730100 }\end{array}$ & {$[8]$} \\
\hline ENSG00000259834 & & $\begin{array}{l}< \\
0.001\end{array}$ & 0.28 & -0.721 & NR & NR & \\
\hline ENSG00000234129 & & 0.001 & 9.68 & 0.272 & NR & NR & \\
\hline ENSG00000269974 & & 0.001 & 2.24 & 0.007 & NR & NR & \\
\hline ENSG00000254887 & LOC100505622 & 0.002 & 0.05 & -0.735 & gastric cancer & $\begin{array}{l}\text { uncharacterized } \\
\text { LOC100505622 }\end{array}$ & {$[12]$} \\
\hline ENSG00000259974 & LINC00261 & 0.003 & 0.69 & -0.187 & $\begin{array}{l}\text { hepatocellular carcinoma, endometrial carcinoma, non-small cell } \\
\text { lung cancer, colon cancer, esiohageal cancer, endometriosis, } \\
\text { choriocarcinoma, gastric cancer, esophageal cancer, lung epithelial } \\
\text { homeostasis, endoderm differentiation }\end{array}$ & $\begin{array}{l}\text { long intergenic } \\
\text { non-protein cod- } \\
\text { ing RNA } 261\end{array}$ & $\begin{array}{l}{[13-19,} \\
25-27]\end{array}$ \\
\hline ENSG00000236366 & LOC153910 & 0.003 & 1.65 & 0.232 & $\begin{array}{l}\text { lung function development, chronic obstructive pulmonary } \\
\text { disease (COPD) and cardiovascular diseases (CVD) }\end{array}$ & $\begin{array}{l}\text { uncharacterized } \\
\text { LOC153910 }\end{array}$ & {$[3,21-24]$} \\
\hline ENSG00000275223 & & 0.003 & 1.56 & 0.024 & NR & NR & \\
\hline ENSG00000253406 & & 0.004 & 967.38 & 1.878 & NR & NR & \\
\hline ENSG00000232079 & LINC01697 & 0.009 & 1.53 & 0.142 & $\begin{array}{l}\text { lung squamous cell carcinoma, gastric cancer, gastric } \\
\text { adenocarcinoma, breast cancer }\end{array}$ & $\begin{array}{l}\text { long intergenic } \\
\text { non-protein cod- } \\
\text { ing RNA } 1697\end{array}$ & [9-11] \\
\hline ENSG00000273837 & & 0.012 & 0.59 & -0.025 & NR & NR & \\
\hline ENSG00000255980 & LOC102724265 & 0.017 & 10.02 & 0.125 & NR & $\begin{array}{l}\text { uncharacterized } \\
\text { LOC102724265 }\end{array}$ & \\
\hline ENSG00000267905 & & 0.018 & 1.5 & 0.032 & NR & NR & \\
\hline ENSG00000176912 & TYMSOS & 0.023 & 1.28 & 0.038 & NR & $\begin{array}{l}\text { TYMS opposite } \\
\text { strand }\end{array}$ & \\
\hline ENSG00000254333 & NDST1-AS1 & 0.032 & 1.21 & 0.044 & NR & $\begin{array}{l}\text { NDST1 antisense } \\
\text { RNA } 1\end{array}$ & \\
\hline ENSG00000231246 & LINC02884 & 0.033 & 0.17 & -0.041 & NR & $\begin{array}{l}\text { long intergenic } \\
\text { non-protein cod- } \\
\text { ing RNA } 2884\end{array}$ & \\
\hline ENSG00000234283 & & 0.047 & 0.39 & -0.459 & NR & NR & \\
\hline
\end{tabular}

NR not reported

${ }^{a}$ Derived from the univariable Cox proportional hazards regression analysis in the 150 training cohort patients

${ }^{\mathrm{b}}$ Derived from the LASSO regression analysis in the 150 training cohort patients

Please refer to the supplementary material for references citation

respectively, except for the lncRNAs (ENSG00000255980, ENSG00000253406, ENSG00000232079, and ENSG00000234283) whose expression showed no significant changes between the low- and high-risk groups (Fig.
S2). Patients in the high-risk group contributed $74.76 \%$ of 1-year recurrence cases and $69.44 \%$ of 2 -year recurrence cases (Fig. 3F). More importantly, correlation assays showed significantly negative correlation of risk score with 1-year 


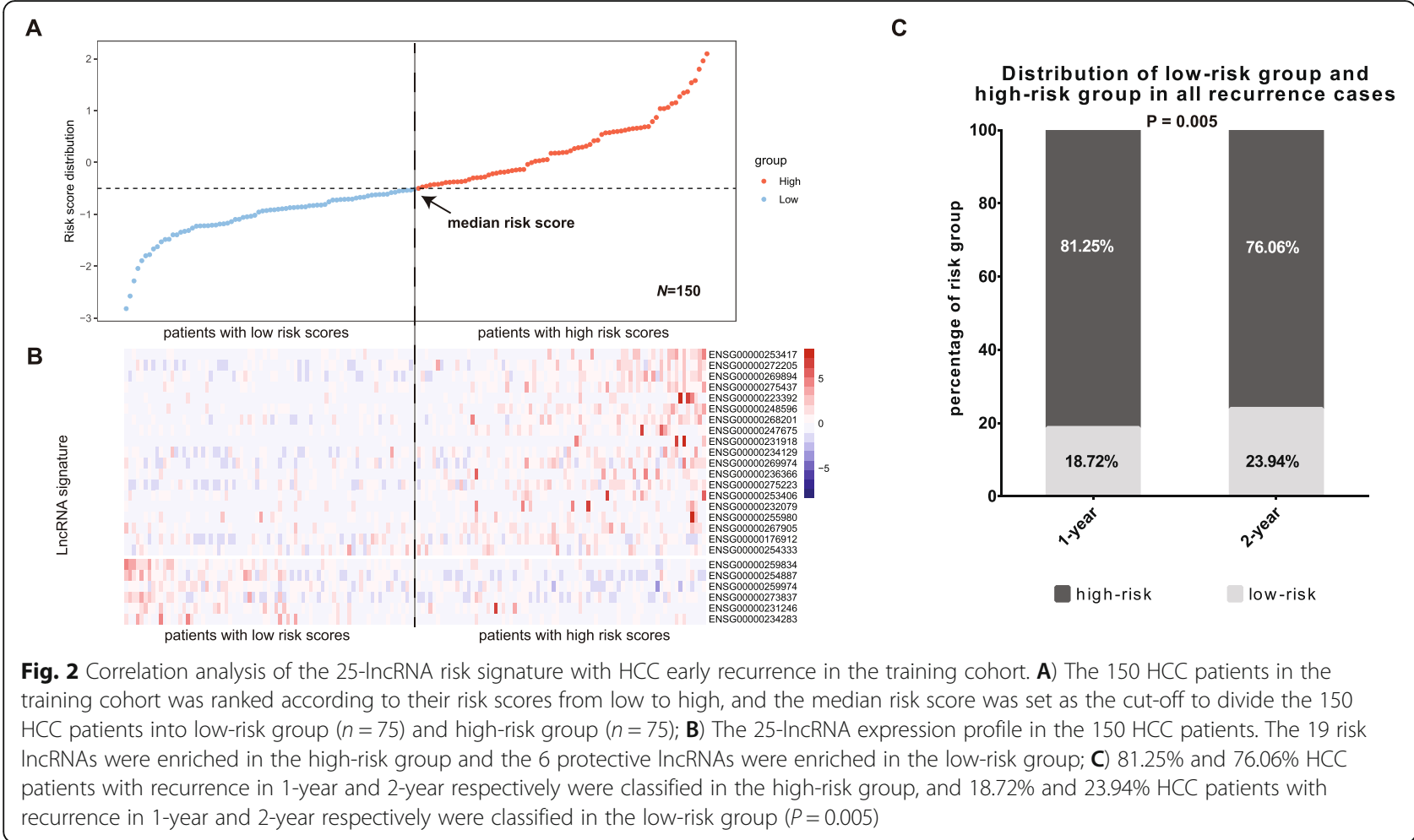

(Fig. 3G) or 2-year DFS (Fig. 3H) in the recurrent HCC patients in the high-risk group. No correlation was observed between risk score and DFS in recurrent HCC patients in the low-risk group (Fig. S3). These findings further validate the correlation of the 25-lncRNA risk signature with HCC early recurrence and indicate the great predictive potential of the risk signature on HCC early recurrence.

The primary purpose for the signature construction study is to accurately discriminate low- and high-risk patients. Therefore, the cut-off selection is critical for the accuracy of the prediction signature. In this study, we adopted the median risk score as cut-off which has been widely employed by many other groups [16, 45-48]. To investigate whether there are other cut-offs which could distinguish low- and high-risk of early recurrence better than the median cut-off, we adopted the cut-off derived from Youden index [49]. Although the Youden index/ cut-off could separate patients into the low- and highrisk groups (Fig. S4A-C), the prediction performance for early recurrence is much poorer than that by using median cut-off (Fig. S4D-F). Therefore, the median risk score used in this study is an appropriate cut-off to accurately distinguish HCC patients with low or high early recurrence risk.

\section{The 25-IncRNA risk signature precisely predicts early recurrence in HCC patients}

To further investigate the prognostic value of the 25lncRNA risk signature for early recurrence, we analyzed cumulative hazard and event in HCC patients. Both cumulative hazards and cumulative events were significantly higher in the high-risk group than those in the low-risk group in either the training cohort (Fig. S5A and B), validation cohort (Fig. S5C and D) or total 299 HCC patients (Fig. S5E and F). Meanwhile, KaplanMeier analyses, in the training cohort (Fig. 4A), validation cohort (Fig. 4B) and 299 enrolled HCC patients (Fig. 4C), showed that the patients in the high-risk group had lower 2-year DFS than those in the low-risk group. These findings further indicate the prognostic value of the 25-lncRNA risk signature for HCC early recurrence.

\section{The 25-IncRNA risk signature is an independent prognostic factor for early recurrence in HCCs}

To determine whether the 25-lncRNA risk signature is an independent prognostic factor for HCC early recurrence, we performed univariate and multivariate Cox regression analyses in the enrolled $299 \mathrm{HCC}$ patients. The 25-IncRNA risk score and other clinicopathological factors, including gender, age, race, cirrhosis, vascular invasion, serum AFP level and TNM stage, were used as covariates. As shown in Table 2, the vascular invasion, serum AFP and 25-IncRNA risk score were significantly associated with 1-year and 2-year recurrence in HCC patients, while the TNM stage was significantly associated with 2-year recurrence but not with 1-year recurrence. These findings are consistent with previous studies showing that serum AFP [50], TNM stage [51] and 


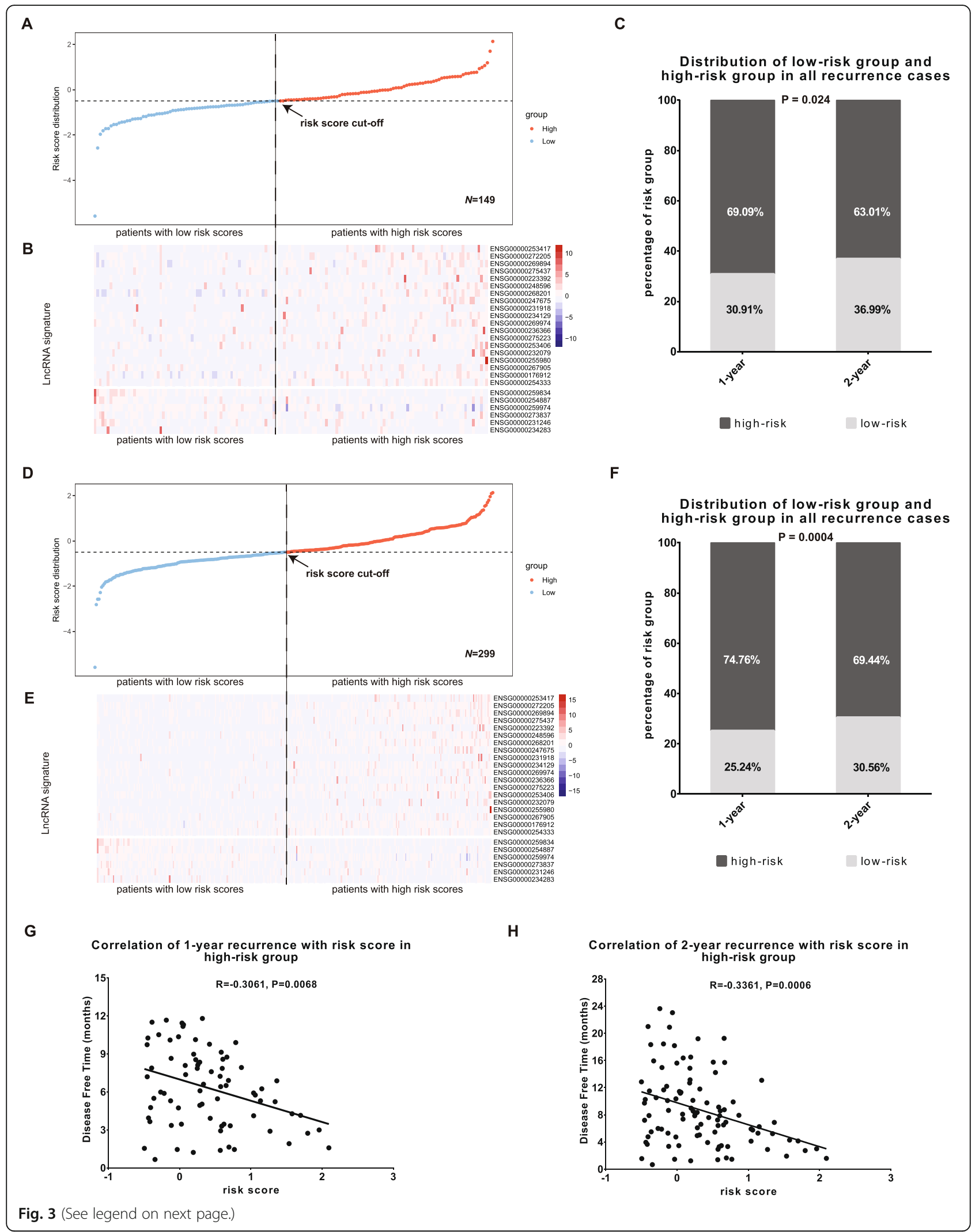


(See figure on previous page.)

Fig. 3 Correlation analysis of the 25-InCRNA risk signature with HCC early recurrence in the validation and entire TCGA cohort. A) The 149 HCC patients in the validation cohort were ranked according to their risk scores from low to high, and divided into the low-risk group $(n=69)$ and the high-risk group ( $n=80$ ) by using the same risk score cut-off in the training cohort; B) The 25-IncRNA expression profile in the 149 HCC patients. The 19 risk IncRNAs were enriched in the high-risk group and the 6 protective IncRNAs were enriched in the low-risk group; C) 69.09\% and 63.01\% HCC patients with recurrence in 1-year and 2-year respectively were classified in the high-risk group, and 30.91\% and 36.99\% HCC patients with recurrence in 1-year and 2-year respectively were assigned in the low-risk group $(P=0.024)$; $\mathbf{D})$ The 299 HCC patients in the entire TCGA cohort were ranked according to their risk scores from low to high, and divided into the low-risk group $(n=144)$ and the high-risk group $(n=155)$ by using the same risk score cut-off in the training cohort; $\mathbf{E})$ The 25 -IncRNA expression profile in the 299 HCC patients. The 19 risk IncRNAs were enriched in the high-risk group and the 6 protective IncRNAs were enriched in the low-risk group; F) $74.46 \%$ and $69.44 \%$ HCC patients with recurrence in 1-year and 2-year respectively were assigned in the high-risk group, and $25.24 \%$ and $30.56 \%$ HCC patients with recurrence in 1-year and 2-year respectively were assigned in the low-risk group $(P=0.0004) ; G$ and $H)$ Correlation of risk score with 1-year (G) or 2-year DFS $(\mathbf{H})$ in the recurrent HCC patients in the high-risk group of the entire TCGA cohort

vascular invasion [2] are independent risk factors for HCC early recurrence, and indicate that the 25-lncRNA risk signature could serve as an independent prognostic factor for HCC early recurrence.

The combination of the 25-IncRNA risk signature, AFP, TNM stage and vascular invasion improves the prognosis evaluation and the construction of nomogram

To investigate which independent risk factor gives the best predictive performance for $\mathrm{HCC}$ early recurrence, ROC analyses were performed by using "pROC". As shown in Fig. 5A and B, the AUC of risk score for 1year recurrence $(73.86 \%)$ and 2-year recurrence $(71.98 \%)$ were better those of AFP (64.58\% for 1-year, $61.39 \%$ for 2 -year recurrence), TNM (64.99\% for 1-year, $67.17 \%$ for 2 -year recurrence) and vascular invasion (VI) (63.47\% for 1 -year, $60.33 \%$ for 2 -year recurrence). Moreover, compared to risk score alone, combining the risk score with AFP, TNM and VI further increased the predictive performance for 1-year recurrence (AUC: $78.79 \%$ vs. 73.86\%) and 2-year recurrence (AUC: $76.82 \%$ vs. $71.98 \%$ ) (Fig. 5C and D). The 95\% confidence interval of AUC and $\mathrm{C}$-index of above signatures were summarized in Table S3. An integrated Nomogram was further constructed by combining the 25-lncRNA signature, AFP, VI and TNM with a C-index 0.739 (Fig. 5E), and the calibration curves of the integrated nomogram for 1year and 2-year DFS were presented in Fig. 5F. Therefore, the combination of the 25-lncRNA risk signature with AFP, TNM and VI could improve the prognosis evaluation for HCC early recurrence.

Biological processes associated with HCC early recurrence Previous studies have shown that lncRNAs function as key regulators of critical biological processes including cell differentiation, development, and apoptosis [52]. To investigate the biological processes associated with HCC early recurrence, gene set enrichment analysis (GSEA) was performed with hallmark pathways based on the gene expression profiling data from HCC patients in the high-risk and the low-risk groups. Eight gene sets were significantly enriched in the high-risk group while no significant gene set enrichment was observed in the lowrisk group $(|\mathrm{NES}|>1$, FDR q-val $<0.25$, NOM p-val $<$ 0.05) (Table 3). Among them, the enrichment of gene sets of "E2F TARGETS", "G2M CHECKPOINT", "MYC TARGETS V1" and "DNA REPAIR" showed higher significance $(|\mathrm{NES}|>1.5$, FDR q-val $<0.10$, NOM p-val $<$
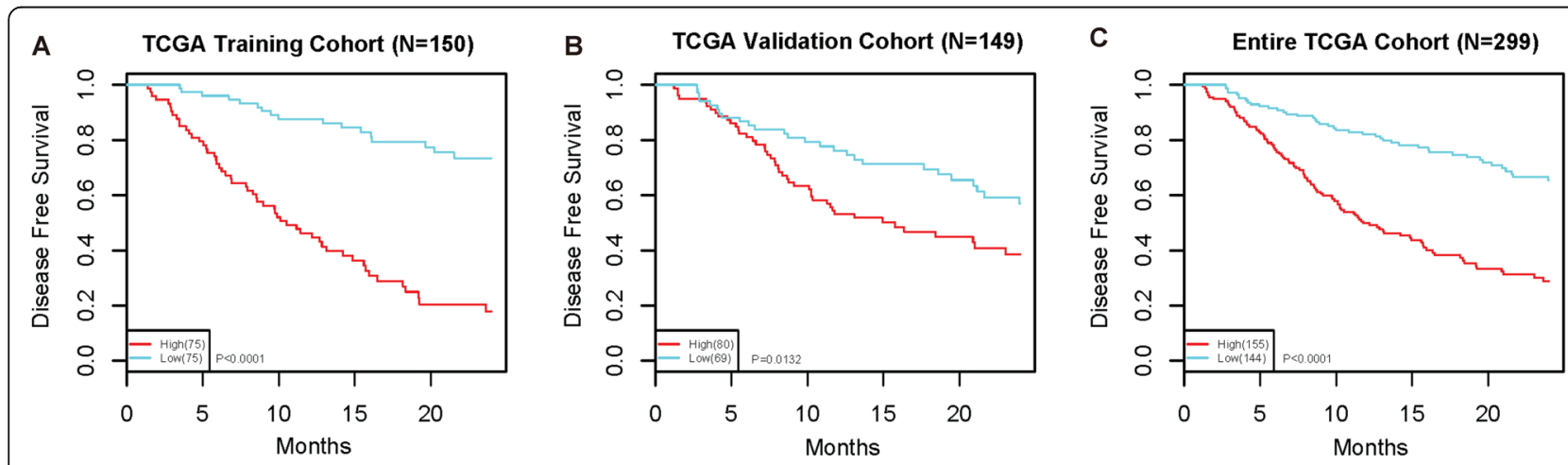

Fig. 4 Kaplan-Meier analysis of the association of the 25-IncRNA risk signature with early recurrence risk in HCCs. A-C) The association of the 25IncRNA risk signature with 2-year DFS was analyzed in the training cohort $(N=150, P<0.0001)(\mathbf{A})$, validation cohort $(N=149, P=0.0132)(\mathbf{B})$, and the entire TCGA cohort $(N=299, P<0.0001)(\mathbf{C})$. The statistical significance was determined by the log-rank test. The patients in each cohort were stratified into the high-risk and low risk groups based on the cut-off risk score in the training cohort 
Table 2 Univariate and multivariate Cox analysis of risk factors in the TCGA entire group $(N=299)$

\begin{tabular}{|c|c|c|c|c|c|c|}
\hline \multirow[t]{2}{*}{ Characteristics } & \multicolumn{3}{|l|}{ Univariate } & \multicolumn{3}{|l|}{ Multivariate } \\
\hline & Hazard Ratio & $\mathrm{Cl} 95$ & $P$ Value & Hazard Ratio & $\mathrm{Cl} 95$ & $P$ Value \\
\hline \multicolumn{7}{|l|}{ 1-year DFS } \\
\hline risk score of 25 -IncRNA signature & 2.38 & $1.88-3.01$ & $<0.001$ & 2 & $1.48-2.71$ & $<0.001$ \\
\hline TNM Stages & 1.96 & $1.55-2.47$ & $<0.001$ & 1.28 & $0.94-1.75$ & 0.124 \\
\hline Vascular Invasion & 1.88 & $1.35-2.61$ & $<0.001$ & 1.66 & $1.12-2.45$ & 0.011 \\
\hline AFP & 2.52 & $1.56-4.07$ & $<0.001$ & 2.23 & $1.34-3.74$ & 0.002 \\
\hline Cirrhosis & 1.11 & $0.65-1.9$ & 0.709 & & & \\
\hline Gender & 1.17 & $0.78-1.77$ & 0.444 & & & \\
\hline Age & 0.77 & $0.49-1.22$ & 0.269 & & & \\
\hline Race & 1.15 & $0.83-1.59$ & 0.396 & & & \\
\hline \multicolumn{7}{|l|}{ 2-year DFS } \\
\hline risk score of 25-IncRNA signature & 2.19 & $1.8-2.66$ & $<0.001$ & 1.92 & $1.48-2.49$ & $<0.001$ \\
\hline TNM Stages & 1.96 & $1.61-2.39$ & $<0.001$ & 1.46 & $1.12-1.89$ & 0.005 \\
\hline Vascular Invasion & 1.8 & $1.36-2.38$ & $<0.001$ & 1.5 & $1.05-2.12$ & 0.024 \\
\hline AFP & 2.03 & $1.37-2.99$ & $<0.001$ & 1.77 & $1.16-2.71$ & 0.008 \\
\hline Cirrhosis & 1.25 & $0.81-1.93$ & 0.305 & & & \\
\hline Gender & 1.21 & $0.86-1.71$ & 0.27 & & & \\
\hline Age & 0.91 & $0.6-1.36$ & 0.635 & & & \\
\hline Race & 0.84 & $0.62-1.12$ & 0.229 & & & \\
\hline
\end{tabular}

In univariate and multivariate Cox analysis, risk score, TNM stages and vascular invasion were evaluated as continuous variables, and AFP, cirrhosis, gender, age and race were evaluated as category variables. Age category and AFP category were defined by 50 and $20 \mathrm{ng} / \mathrm{ml}$ as cut-off value, respectively

0.01) (Table 3). The snapshots of enrichment results were displayed in Fig. 6 and the heatmaps for enriched gene sets were displayed in Fig. S6. These findings suggest that the 25 IncRNAs may affect HCC early recurrence through E2F, Myc, G2M and DNA repair pathways.

\section{The 25-IncRNA signature negatively associates with tumor infiltrating lymphocytes}

Tumor infiltrating lymphocytes (TILs) have been recognized as a prognostic factor in various types of cancers, and accumulation of TILs has been established as a positive prognostic factor in a number of solid cancers including melanoma [53], colon cancer [54] and ovarian cancer [55]. Previous studies have demonstrated that HCC patients with prominent TILs showed reduced recurrence and better prognosis compared with those without prominent TILs [56, 57]. Although the TILs are minority in the tumor bulk, the immune checkpoint molecules specifically express on $\mathrm{T}$ cells and antigen presenting cells but not tumor cells or other stromal cells in tumor bulk. Thus, it is a commonly accepted approach to evaluate TILs by using the expression levels of immune checkpoint molecules from bulk-tumor data $[45,58,59]$. To investigate whether the 25-IncRNA prognostic signature could reflect the levels of TILs, comparison of TILs was performed between the low- and high-risk groups. As shown in Fig. 7A, 22 out of 28 TILs showed significant enrichment in the low-risk group compared to the high-risk group $(P<0.05)$. Correlation analysis between risk scores and normalized enrichment scores (NES) of TILs revealed that the intratumor accumulation of 23 TILs was negatively associated with risk scores $(P<0.05$, Fig. $7 \mathrm{~B})$. Among them, the type $1 \mathrm{~T}$ helper cell, effector memory CD8 $\mathrm{T}$ cell and activated CD8 $\mathrm{T}$ cell, which are well-known antitumor immune cells, ranked as the top 3 TILs negatively associated with the risk scores ( $|\mathrm{NES}|>0.4$, Fig. 7C-E). These findings suggested that the 25-lncRNA prognostic signature may reflect the levels of TILs and predict the postsurgery prognosis in HCCs.

The low-risk group patients showed more sensitivity in immunotherapies and the low- and high-risk group patients showed different chemotherapies responses

Since more TILs significantly enriched in the low-risk group patients, we attempted to further investigate whether the immunotherapies response are different in the low- and high-risk group. TIDE prediction suggest that there was no significantly difference in immunotherapies response between the low- $(48.61 \%, 70 / 144)$ and high-risk $(42.58 \%, 66 / 155)$ group $(P=0.297)$. However, by mapping the expression profile of the low- and high-risk group with a public dataset of 47 melanoma 

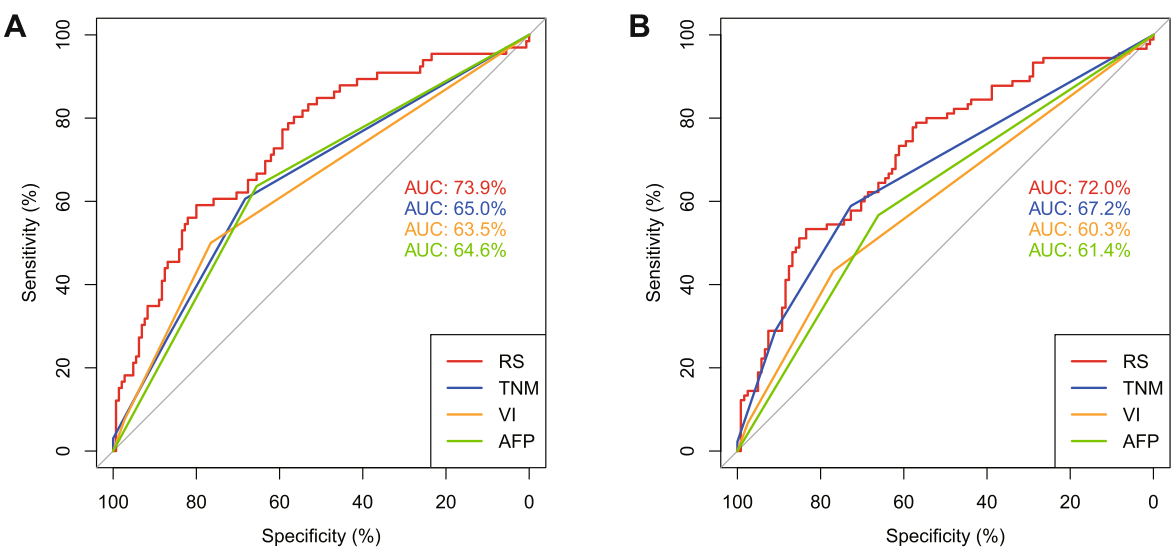

C

D
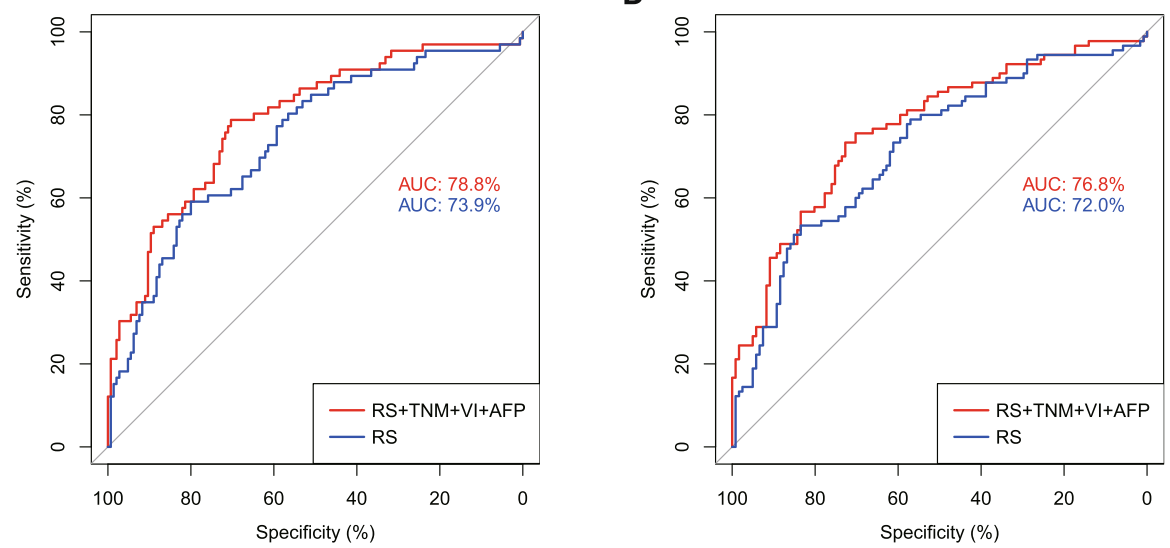

E

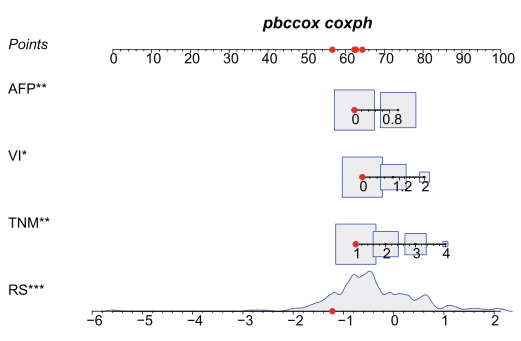

Total points

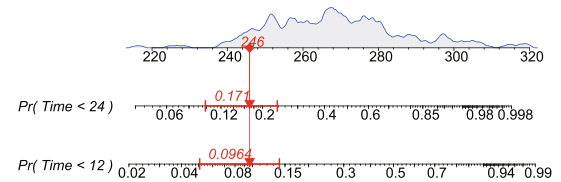

$\mathbf{F}$

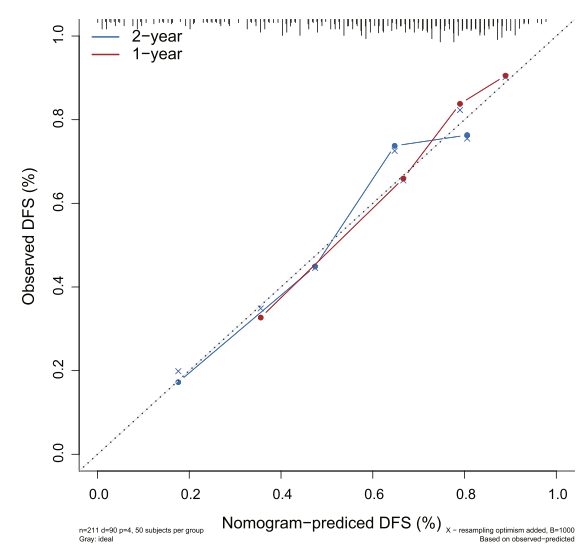

Fig. $5 \mathrm{ROC}$ analysis of the predictive performance and nomogram construction for early recurrence of the 25-IncRNA risk signature, TNM stage, vascular invasion and AFP. A-B) ROC analysis of the predictive performance of the 25-IncRNA risk signature, TNM stages, vascular invasion and AFP for 1-year DFS (A) and 2-year DFS (B) in the entire TCGA cohort; C-D) ROC analysis of the predictive performance of the combination of the 25-IncRNA risk signature, TNM stages, vascular invasion and AFP and risk score alone for 1-year DFS (C) and 2-year DFS (D) in the entire TCGA cohort. E) Nomogram of the 25-IncRNA signature risk score combined with AFP, vascular invasion and TNM stages; F) Calibration curves for the 25-IncRNA-signature-integrated nomogram for 1-year DFS and 2-year DFS. RS: the risk score of the 25-IncRNA signature, VI: vascular invasion

patients responded to immunotherapies in SubMap modules of GenePattern [60], the low-risk group showed prospective response to anti-PD-1 (programmed cell death protein 1 ) therapy (Bonferroni-corrected $P=0.008$, Fig. 8A). Besides immunotherapies, we attempted to identify whether the 25-lncRNA prognostic signature could be applied to chemotherapies prediction. The results showed that the low-risk group had a lower half maximal inhibitory concentration of docetaxel, gefitinib and vinblastine, while the high-risk group had a lower 
Table 3 GSEA pathways up-regulated in high-risk group

\begin{tabular}{|c|c|c|c|c|}
\hline Gene Sets & SIZE & NES & NOM p-val & FDR q-val \\
\hline HALLMARK_E2F_TARGETS & 195 & 1.991 & 0.000 & 0.045 \\
\hline HALLMARK_G2M_CHECKPOINT & 189 & 1.845 & 0.002 & 0.077 \\
\hline HALLMARK_MYC_TARGETS_V1 & 194 & 1.844 & 0.004 & 0.052 \\
\hline HALLMARK_DNA_REPAIR & 148 & 1.826 & 0.004 & 0.045 \\
\hline HALLMARK_MYC_TARGETS_V2 & 58 & 1.641 & 0.040 & 0.153 \\
\hline HALLMARK_UNFOLDED_PROTEIN_RESPONSE & 107 & 1.562 & 0.043 & 0.207 \\
\hline HALLMARK_MITOTIC_SPINDLE & 198 & 1.542 & 0.019 & 0.199 \\
\hline HALLMARK_GLYCOLYSIS & 198 & 1.516 & 0.022 & 0.205 \\
\hline
\end{tabular}

half maximal inhibitory concentration of doxorubicin, mitomycin $\mathrm{C}$ and paclitaxel (Fig. $8 \mathrm{~B}, P<0.05$ ). Thus, the 25-IncRNA prognostic signature could act as a potential predictor for immunotherapies and chemotherapies.

\section{Discussion}

As a class of non-coding transcripts, lncRNAs have been identified in all model organisms. So far, over 56,000 human lncRNAs have been reported in recent lncRNA annotations and the number of lncRNAs keeps growing [61]. Unlike protein-coding genes, most lncRNAs are less conserved, which leads to neglect of the function of IncRNAs [62]. However, accumulating evidence mainly at cellular level has indicated the involvement of lncRNAs in various biological processes such cell proliferation, apoptosis and nutrient sensing to cell differentiation [63]. Moreover, dysregulation of lncRNAs has been implicated in the pathogenesis of various diseases including cancers [64]. Many lncRNAs have shown their prognostic value in many types of cancers [26, 27]. In this study, we established a 25-lncRNA risk signature to predict HCC early recurrence. We demonstrated that, compared to AFP, TNM and VI, this 25-lncRNA risk signature possesses the best prognostic potential for $\mathrm{HCC}$ early recurrence. Moreover, the combination of the lncRNA risk signature with AFP, TNM and VI could further improve the predictive performance.

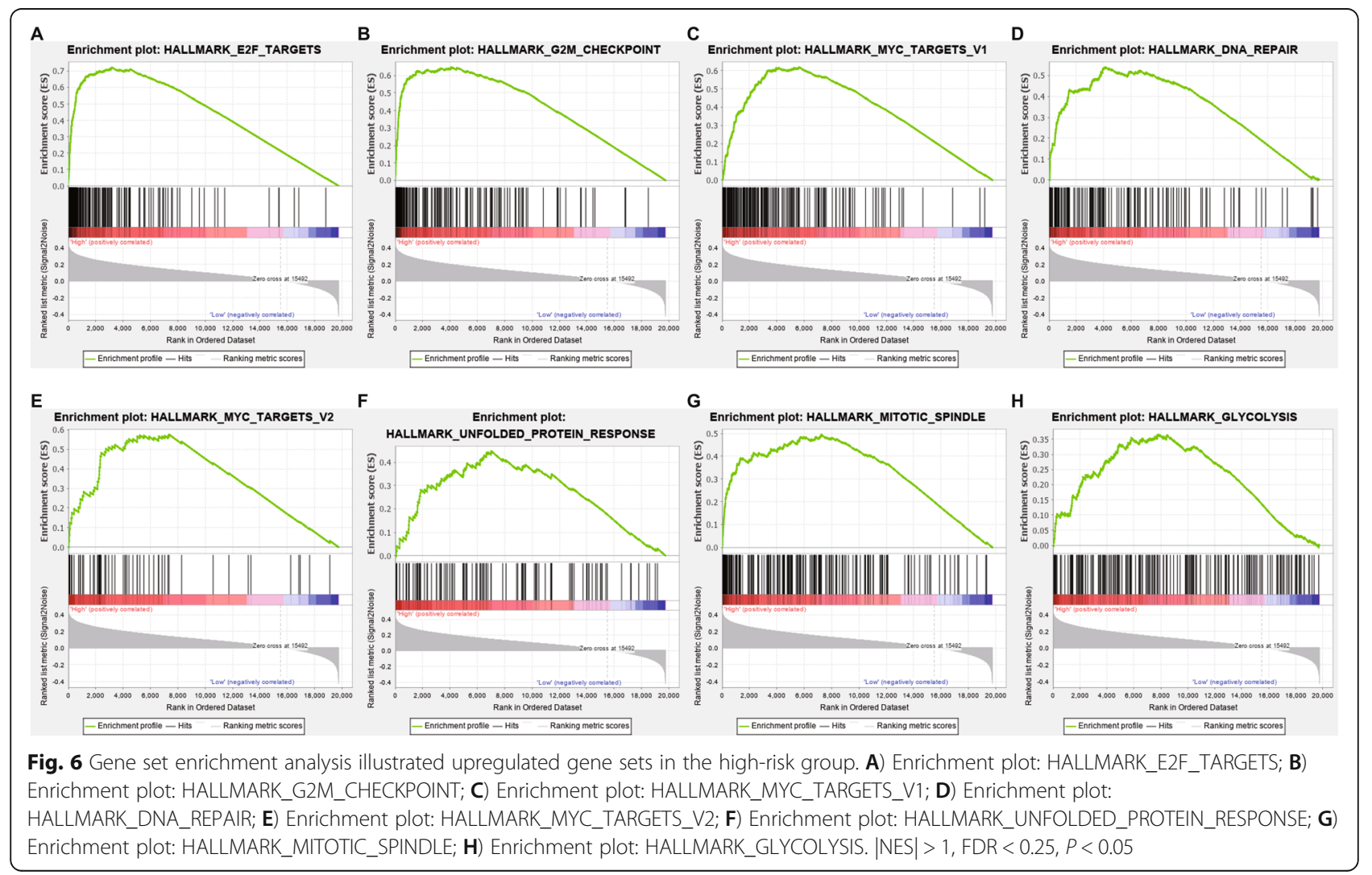



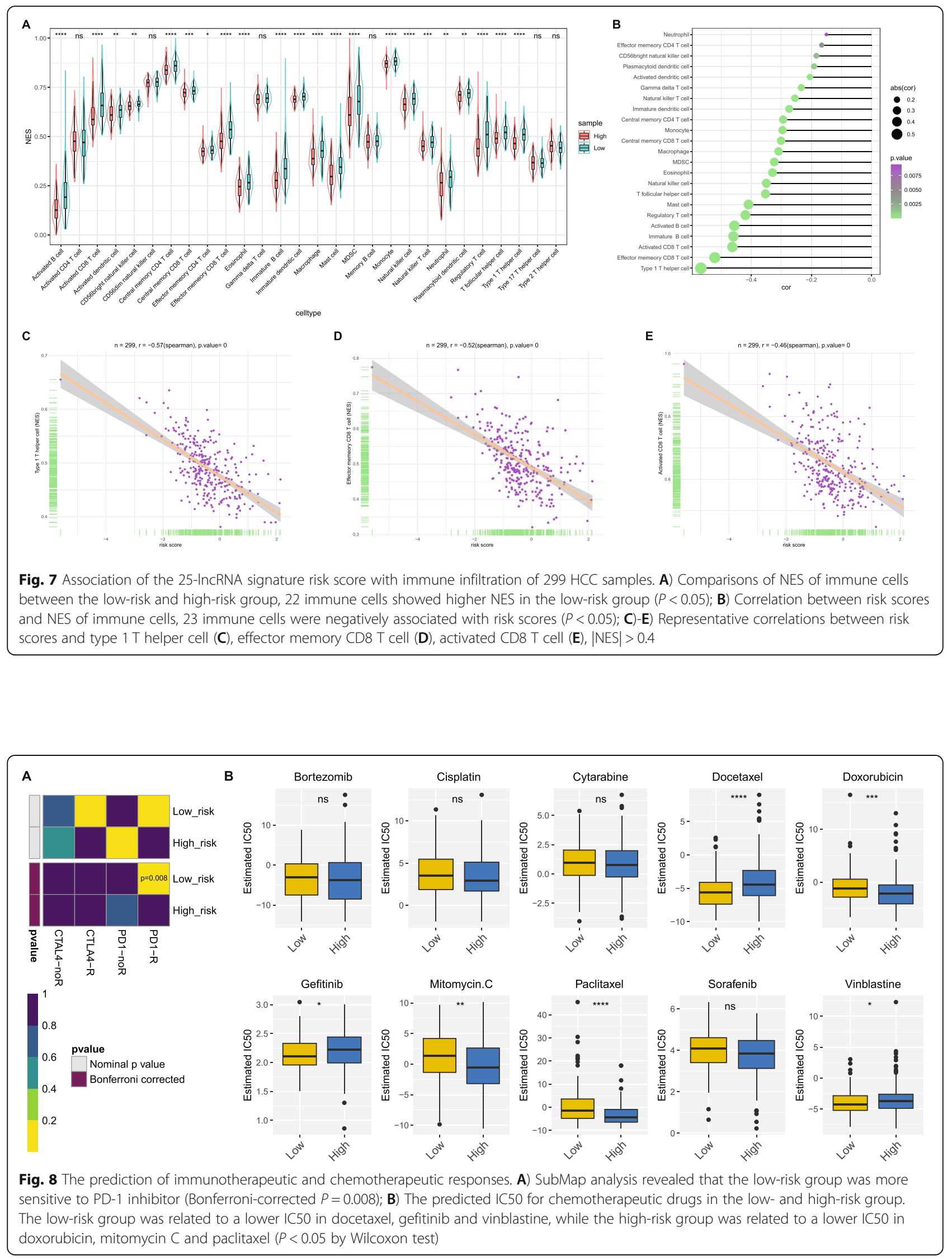
In this study, we define the recurrence in 2-year postsurgery as HCC early recurrence. This is in agreement with a previous study showing that the slopes of early recurrence curve and late recurrence curve are different and the intercept time point of the two curves is defined as the cut-off to separate early and late recurrence [4]. This separation criterion is widely adopted by many other studies [65]. We also noticed that some studies define the recurrence in 1-year post-surgery as HCC early recurrence $[66,67]$. Therefore, we analyzed the association of the 25-lncRNA risk signature with both 1-year and 2-year recurrence in most of our analyses and found that this risk signature has great prognostic potential for both of them.

The 25-lncRNA risk signature includes 19 risk lncRNAs (coefficient $>0$ ) and 6 protective lncRNAs (coefficient $<0$ ) (Table 1). Among these lncRNAs, dysregulation of LINC02159, CLDN10-AS1, LOC643201, LRP4AS1, LOC730100, LINC01697, LOC100505622, and LINC00261 has been reported in several types of cancers (Table 1). In addition, CLDN10-AS1 was reported to be involved in endothelial dysfunction in atherogenesis (Table 1). Some previous studies have suggested the association of LOC153910 with lung function development, risk of chronic obstructive pulmonary disease (COPD) and cardiovascular diseases (CVD) (Table 1). LINC00261 has shown to regulate endoderm differentiation, lung epithelial homeostasis and endometriosis (Table 1). Given the fact that most of IncRNAs demonstrate a tissue-specific expression pattern [68], further investigation of the role of those 25 lncRNAs in HCCs is warranted.

To investigate the biological processes or pathways related to HCC early recurrence, we performed GSEA to explore the hallmarks of gene sets in the high-risk group. Total 8 gene sets were significantly enriched in the high-risk group. Among them, the gene sets of "E2F TARGETS", "G2M CHECKPOINT", "MYC TARGETS V1" and "DNA REPAIR" showed higher significance in enrichment. In fact, members of those four gene sets have been reported to associate with poor prognosis in many types of cancers including HCC [69-72].

Accumulation of TILs is commonly related to an improved prognosis in many types of cancers. In the present study, greater intratumor accumulation of TILs was observed in the low-risk group compared to highrisk group. We demonstrated that the 25-signature risk score significantly and negatively associate with intratumor accumulation of type $1 \mathrm{~T}$ helper cell, effector memory CD8 $\mathrm{T}$ cell and activated CD8 $\mathrm{T}$ cell, which are well-known antitumor immune cells involved in cancer immune therapy [73-75], further suggesting that this 25-IncRNA signature has potential to predict the postsurgery prognosis in HCC patients. In addition, the immunotherapies prediction based on this 25-lncRNA signature suggested that the low-risk group had more effective response to PD-1 inhibitor. Moreover, chemotherapies prediction indicated that the low- and highrisk showed different sensitivity to drugs such as docetaxel and paclitaxel, but not cisplatin and sorafenib. Thus, different therapies might be adapted to HCC patient in the low- and high risk group according to the 25-IncRNA signature.

Although the 25-lncRNA risk signature was validated in the TCGA internal validation cohort and displayed good prognostic potential in the enrolled 299 HCC patients, an external validation cohort is missing in this study. This is because we failed to find any suitable GEO datasets or International Cancer Genome Consortium (ICGC) database which could apply sufficient information on both lncRNA expression profile and clinical survival. For example, there are two GEO datasets, GSE67260 and GSE113850, possess satisfied data on lncRNA expression profile but without clinical records. Moreover, expression profiles of 22 lncRNAs in the 25lncRNA signature could be extracted from two ICGC datasets including LIRI-JP and LICA-FR, but disease free survival information is missing. The Cancer Genome Atlas (TCGA) is a multi-institutional, cross-discipline effort led by the National Cancer Institute recruiting cancer samples from different countries. For example, the HCC samples recruited in the TCGA-LIHC database were derived from Vietnam, United States, Canada, South Korea, Russia [76]. Therefore, those samples are actually derived from multi-centers and at certain level support the approach we used in this study by splitting them into a training cohort and a validation cohort. However, validation of this 25-lncRNA risk signature in an external cohort will be warranted as long as suitable data are available. Meanwhile, the validation of individual lncRNA included in this 25-lncRNA signature in clinical HCC tumor and paracancerous tissues were in processing but have not completed yet. LncRNAs ENSG00000231918, ENSG00000248596, and ENSG00000223392 were found to be upregulated in 36 HCC tumor tissues compared with paracancerous tissues (Fig. S7, Table S4).

\section{Conclusions}

In this study, we established a 25-lncRNA risk signature for HCC early recurrence. According to this risk signature, HCC patients could be accurately separated into the low- and high-risk groups. 1-year and 2-year recurrence rates were significantly higher in the high-risk group than those in the low-risk group. More importantly, the risk score significantly and negatively correlates with DFS in recurrent HCC patients in the highrisk group. Univariate and multivariate Cox regression 
analyses showed that the 25-lncRNA risk score, serum AFP, TNM stage and vascular invasion (VI) were independent prognostic factors for HCC early recurrence. Moreover, compared to serum AFP, TNM stage and VI, the 25-lncRNA risk signature showed better prognostic potential for HCC early recurrence. In addition, the combination of the 25-lncRNA risk signature with serum AFP, TNM stage and VI could further improve the prognostic potential for $\mathrm{HCC}$ early recurrence. Meanwhile, GSEA showed that several gene sets related to malignancy, such as "E2F TARGETS", "G2M CHECKPOINT", "MYC TARGETS V1" and "DNA REPAIR", have significantly enriched in the high-risk group, suggesting that lncRNAs included in this risk signature may affect HCC progression through those biological pathways. Moreover, ssGSEA revealed greater TILs in the low-risk group compared to the high-risk group and the negative association between the 25-lncRNA risk signature score and the intratumor population of several key antitumor TILs such as type $1 \mathrm{~T}$ helper cell, effector memory CD8 $\mathrm{T}$ cell and activated CD8 T cell, and SubMap algorithm predicted that the low-risk group was more sensitive to anti-PD-1 therapy. Finally, Chemotherapies prediction revealed that the low risk was associated with sensitivity to docetaxel, gefitinib and vinblastine, while high risk was associated with sensitivity to doxorubicin, mitomycin $\mathrm{C}$ and paclitaxel.

\footnotetext{
Abbreviations

HCC: Hepatocellular carcinoma; IncRNA: long non-coding RNA; TCGA: The Cancer Genome Atlas; DEG: Differentially expressed gene; LASSO: Least absolute shrinkage and selection operator; ROC: Receiver operating characteristic curve; GSEA: Gene set enrichment analysis; TILs: Tumor infiltrating lymphocytes; ssGSEA: single sample Gene Set Enrichment Analysis; GDSC: Genomics of Drug Sensitivity in Cancer; AJCC: American Joint Committee on Cancer; BCLC: Barcelona Clinic Liver Cancer; CLIP: Cancer of the Liver Italian Program; AFP: Alpha-fetoprotein; FDA: Food and Drug Administration; CRC: Colorectal cancer; LIHC: Liver Hepatocellular Carcinoma; OS: Overall survival; DFS: Disease free survival; ER IncRNAs : IncRNAs associated with HCC early recurrence; VI: Vascular invasion; NES: Normalized enrichment scores; PD-1: Programmed cell death protein 1; COPD: Chronic obstructive pulmonary disease; CVD: Cardiovascular diseases;

ICGC: International Cancer Genome Consortium
}

\section{Supplementary Information}

The online version contains supplementary material available at https://doi. org/10.1186/s12885-021-08827-z.

\section{Additional file 1.}

\section{Acknowledgements}

Not applicable.

\section{Authors' contributions}

Yi Fu, and Hailong Wu designed the project. Yi Fu, Xindong Wei and Yuhui Xu performed bioinformatics and statistical analyses. Xindong Wei and Xinjie Ling performed the experiments. Yi Fu, Qiuqin Han, Jiamei Le, Yujie Ma, Yuhui Xu, Ning Liu, Xuan Wang and Ying Tong performed extensive literature search and discussion. Yi Fu, and Hailong Wu drafted the manuscript. Hailong Wu, Xiaoni Kong and Jinyang Gu edited the manuscript. All authors read and approved the final manuscript.

\section{Funding}

This work was supported by grants from the National Natural Science Foundation of China (31671453, 31870905 to Hailong Wu, 81772507 to Jinyang Gu), the Scientific Program of Shanghai Municipal Health Commission (SHWJ2019211 to Hailong Wu), Zhejiang Medical and Health Science and Technology Project (No.: WKJ2009-2-036), Construction Project of Shanghai Key Laboratory of Molecular Imaging (18DZ2260400), Shanghai Municipal Education Commission (Class II Plateau Disciplinary Construction Program of Medical Technology of SUMHS, 2018-2020), the Key Program of National Natural Science Foundation of China (Grant No.81830052), the Hundred Teacher Talent Program (B3-0200-20-311008-23 to Yi Fu) and the University-level Scientific Fund (E3-0200-21-201011-42 to Yi Fu) of Shanghai University of Medicine and Health Sciences.

\section{Availability of data and materials}

The dataset supporting the conclusions of this article is available in the TCGA-LIHC repository, http://cancergenome.nih.gov/.

\section{Declarations}

\section{Ethics approval and consent to participate}

The experimental protocol was established, according to the ethical guidelines of the Helsinki Declaration and was approved by the Human Ethics Committee of Shanghai University of Medicine \& Health Sciences. Written informed consent was obtained from individual or guardian participants.

\section{Consent for publication}

Not applicable.

\section{Competing interests}

The authors declare that they have no competing interests.

\section{Author details}

${ }^{1}$ Affiliated Zhoupu Hospital, Shanghai University of Medicine and Health Sciences, Shanghai 201318, China. ${ }^{2}$ Shanghai Key Laboratory of Molecular Imaging, Collaborative Innovation Center for Biomedicines, Shanghai University of Medicine and Health Sciences, Shanghai 201318, China. ${ }^{3}$ School of Medical Instruments, Shanghai University of Medicine and Health Sciences, Shanghai 201318, China. ${ }^{4}$ Nanjing University of Traditional Chinese Medicine, Nanjing 210000, China. ${ }^{5}$ Graduate School of Art and Sciences, Columbia University, New York, NY 10027, USA. ${ }^{6}$ Department of Clinical Oncology, Taian City Central Hospital, Taian 271000, Shandong, China. ${ }^{7}$ Department of General Surgery, Nanjing General Hospital of Nanjing Military Command, Nanjing 210000, China. ${ }^{8}$ Institute of Clinical Immunology, Department of Liver Diseases, Central Laboratory, Shuguang Hospital Affiliated to Shanghai University of Traditional Chinese Medicine, Shanghai 200021, China. ${ }^{9}$ Department of Transplantation, Xinhua Hospital Affiliated to Shanghai Jiao Tong University School of Medicine, Shanghai 200092, China.

${ }^{10}$ Department of Liver Surgery, Renji Hospital Affiliated to Shanghai Jiao

Tong University School of Medicine, Shanghai 200127, China.

Received: 26 April 2021 Accepted: 30 September 2021

Published online: 30 October 2021

References

1. Bray F, Ferlay J, Soerjomataram I, Siegel RL, Torre LA, Jemal A. Global cancer statistics 2018: GLOBOCAN estimates of incidence and mortality worldwide for 36 cancers in 185 countries. CA Cancer J Clin. 2018;68(6):394-424. https://doi.org/10.3322/caac.21492.

2. Shah SA, Cleary SP, Wei AC, Yang I, Taylor BR, Hemming AW, et al. Recurrence after liver resection for hepatocellular carcinoma: risk factors, treatment, and outcomes. Surgery. 2007;141(3):330-9. https://doi.org/10.101 6/j.surg.2006.06.028.

3. Sherman M. Recurrence of hepatocellular carcinoma. N Engl J Med. 2008; 359(19):2045-7. https://doi.org/10.1056/NEJMe0807581.

4. Portolani N, Coniglio A, Ghidoni S, Giovanelli M, Benetti A, Tiberio GA, et al. Early and late recurrence after liver resection for hepatocellular carcinoma: prognostic and therapeutic implications. Ann Surg. 2006;243(2):229-35. https://doi.org/10.1097/01.sla.0000197706.21803.a1. 
5. Liu P-H, Hsu C-Y, Hsia C-Y, Lee Y-H, Su C-W, Huang Y-H, et al. Prognosis of hepatocellular carcinoma: assessment of eleven staging systems. J Hepatol. 2016;64(3):601-8. https://doi.org/10.1016/j.jhep.2015.10.029.

6. Zhu J, Tang B, Li J, Shi Y, Chen M, Lv X, et al. Identification and validation of the angiogenic genes for constructing diagnostic, prognostic, and recurrence models for hepatocellular carcinoma. Aging (Albany NY). 2020; 12(9):7848-73. https://doi.org/10.18632/aging.103107.

7. Marrero JA, Kulik LM, Sirlin CB, Zhu AX, Finn RS, Abecassis MM, et al. Diagnosis, staging, and management of hepatocellular carcinoma: 2018 practice guidance by the American Association for the Study of Liver Diseases. Hepatology. 2018;68(2):723-50.

8. van 't Veer LJ, Dai H, van de Vijver MJ, He YD, Hart AA, Mao M, et al. Gene expression profiling predicts clinical outcome of breast cancer. Nature. 2002 415(6871):530-6. https://doi.org/10.1038/415530a.

9. van de Vijver MJ, He YD, van't Veer LJ, Dai H, Hart AA, Voskuil DW, et al. A gene-expression signature as a predictor of survival in breast cancer. N Engl J Med. 2002;347(25):1999-2009. https://doi.org/10.1056/NEJMoa021967.

10. Esserman $\sqcup$, Yau C, Thompson CK, van 't Veer L, Borowsky AD, Hoadley KA, et al. Use of molecular tools to identify patients with indolent breast cancers with ultralow risk over 2 decades. JAMA Oncol. 2017;3(11):1503-10. https://doi.org/10.1001/jamaoncol.2017.1261.

11. Buyse M, Loi S, van't Veer L, Viale G, Delorenzi M, Glas AM, et al. Validation and clinical utility of a 70-gene prognostic signature for women with nodenegative breast cancer. J Natl Cancer Inst. 2006;98(17):1183-92. https://doi. org/10.1093/jnci/djj329.

12. Mook S, Schmidt MK, Weigelt B, Kreike B, Eekhout I, van de Vijver MJ, et al. The 70-gene prognosis signature predicts early metastasis in breast cancer patients between 55 and 70 years of age. Ann Oncol. 2010;21(4):717-22. https://doi.org/10.1093/annonc/mdp388.

13. Salazar R, Roepman P, Capella G, Moreno V, Simon I, Dreezen C, et al. Gene expression signature to improve prognosis prediction of stage II and III colorectal cancer. J Clin Oncol. 2011;29(1):17-24. https://doi.org/10.1200/ JCO.2010.30.1077.

14. Maak M, Simon I, Nitsche U, Roepman P, Snel M, Glas AM, et al. Independent validation of a prognostic genomic signature (ColoPrint) for patients with stage II colon cancer. Ann Surg. 2013;257(6):1053-8. https:// doi.org/10.1097/SLA.0b013e31827c1180.

15. Kopetz S, Tabernero J, Rosenberg R, Jiang Z-Q, Moreno V, BachleitnerHofmann T, et al. Genomic classifier ColoPrint predicts recurrence in stage II colorectal cancer patients more accurately than clinical factors. Oncologist. 2015;20(2):127-33. https://doi.org/10.1634/theoncologist.2014-0325.

16. Wei R, Huang GL, Zhang MY, Li BK, Zhang HZ, Shi M, et al. Clinical significance and prognostic value of microRNA expression signatures in hepatocellular carcinoma. Clin Cancer Res. 2013;19(17):4780-91. https://doi. org/10.1158/1078-0432.CCR-12-2728.

17. Nault JC, De Reynies A, Villanueva A, Calderaro J, Rebouissou S, Couchy G, et al. A hepatocellular carcinoma 5-gene score associated with survival of patients after liver resection. Gastroenterology. 2013;145(1):176-87. https:// doi.org/10.1053/j.gastro.2013.03.051

18. Kim JH, Sohn BH, Lee HS, Kim SB, Yoo JE, Park YY, et al. Genomic predictors for recurrence patterns of hepatocellular carcinoma: model derivation and validation. PLoS Med. 2014;11(12):e1001770. https://doi.org/10.1371/journal. pmed. 1001770.

19. Bai Y, Lin H, Chen J, Wu Y, Yu S. Identification of prognostic glycolysisrelated IncRNA signature in tumor immune microenvironment of hepatocellular carcinoma. Front Mol Biosci. 2021;8:645084. https://doi.org/1 0.3389/fmolb.2021.645084

20. Weng J, Zhou C, Zhou Q, Chen W, Yin Y, Atyah M, et al. Development and validation of a metabolic gene-based prognostic signature for hepatocellular carcinoma. J Hepatocell Carcinoma. 2021;8:193-209. https:/ doi.org/10.2147/JHC.S300633

21. Deng X, Bi Q, Chen S, Chen X, Li S, Zhong Z, et al. Identification of a fiveautophagy-related-IncRNA signature as a novel prognostic biomarker for hepatocellular carcinoma. Front Mol Biosci. 2020;7:611626. https://doi.org/1 0.3389/fmolb.2020.611626.

22. Okazaki Y, Furuno M, Kasukawa T, Adachi J, Bono H, Kondo S, et al. Analysis of the mouse transcriptome based on functional annotation of 60,770 full-length cDNAs. Nature. 2002;420(6915):563-73. https://doi.org/10.1038/nature01266.

23. Shi $X$, Sun M, Liu H, Yao Y, Song Y. Long non-coding RNAs: a new frontier in the study of human diseases. Cancer Lett. 2013;339(2):159-66. https://doi. org/10.1016/j.canlet.2013.06.013.
24. Huang MD, Chen WM, Qi FZ, Xia R, Sun M, Xu TP, et al. Long non-coding RNA ANRIL is upregulated in hepatocellular carcinoma and regulates cell apoptosis by epigenetic silencing of KLF2. J Hematol Oncol. 2015;8(50):1-14.

25. Malakar P, Shilo A, Mogilevsky A, Stein I, Pikarsky E, Nevo Y, et al. Long noncoding RNA MALAT1 promotes hepatocellular carcinoma development by SRSF1 upregulation and mTOR activation. Cancer Res. 2017;77(5):115567. https://doi.org/10.1158/0008-5472.CAN-16-1508.

26. Yuan S-X, Yang F, Yang Y, Tao Q-F, Zhang J, Huang G, et al. Long noncoding RNA associated with microvascular invasion in hepatocellular carcinoma promotes angiogenesis and serves as a predictor for hepatocellular carcinoma patients' poor recurrence-free survival after hepatectomy. Hepatology (Baltimore, Md). 2012;56(6):2231-41.

27. Huang J-L, Cao S-W, Ou Q-S, Yang B, Zheng S-H, Tang J, et al. The long non-coding RNA PTTG3P promotes cell growth and metastasis via upregulating PTTG1 and activating PI3K/AKT signaling in hepatocellular carcinoma. Mol Cancer. 2018;17(1):93. https://doi.org/10.1186/s12943-0180841-x.

28. Robinson MD, McCarthy DJ, Smyth GK. edgeR: a Bioconductor package for differential expression analysis of digital gene expression data. Bioinformatics. 2010;26(1):139-40. https://doi.org/10.1093/bioinformatics/ btp616.

29. McCarthy DJ, Chen Y, Smyth GK. Differential expression analysis of multifactor RNA-Seq experiments with respect to biological variation. Nucleic Acids Res. 2012;40(10):4288-97. https://doi.org/10.1093/nar/gks042.

30. Therneau TM, Grambsch PM. Modeling survival data: extending the Cox model. New York: Springer; 2000. https://doi.org/10.1007/978-1-4757-3294-8.

31. Pathan M, Keerthikumar S, Ang C-S, Gangoda L, Quek CYJ, Williamson NA, et al. FunRich: an open access standalone functional enrichment and interaction network analysis tool. Proteomics. 2015;15(15):2597-601. https:// doi.org/10.1002/pmic.201400515

32. Simon N, Friedman J, Hastie T, Tibshirani R. Regularization paths for Cox's proportional hazards model via coordinate descent. J Stat Softw. 2011;39(5): 1-13. https://doi.org/10.18637/jss.v039.105.

33. Tibshirani R, Bien J, Friedman J, Hastie T, Simon N, Taylor J, et al. Strong rules for discarding predictors in lasso-type problems. J R Stat Soc Series B Stat Methodol. 2012;74(2):245-66. https://doi.org/10.1111/j.1467-9868.2011.01004.x.

34. Robin X, Turck N, Hainard A, Tiberti N, Lisacek F, Sanchez J-C, et al. pROC: an open-source package for $\mathrm{R}$ and $\mathrm{S}+$ to analyze and compare ROC curves. BMC Bioinformatics. 2011;12(1):77. https://doi.org/10.1186/1471-2105-12-77.

35. Marshall R. regplot: Enhanced Regression Nomogram Plot. R package version 1.12020 [Available from: https://CRAN.R-project.org/package= regplot.

36. Hanzelmann S, Castelo R, Guinney J. GSVA: gene set variation analysis for microarray and RNA-seq data. BMC Bioinformatics. 2013;14(1):7. https://doi. org/10.1186/1471-2105-14-7.

37. Charoentong P, Finotello F, Angelova M, Mayer C, Efremova M, Rieder D, et al. Pan-cancer Immunogenomic analyses reveal genotypeImmunophenotype relationships and predictors of response to checkpoint blockade. Cell Rep. 2017;18(1):248-62. https://doi.org/10.1016/j.celrep.201 6.12 .019 .

38. Jiang P, Gu S, Pan D, Fu J, Sahu A, Hu X, et al. Signatures of T cell dysfunction and exclusion predict cancer immunotherapy response. Nat Med. 2018;24(10):1550-8. https://doi.org/10.1038/s41591-018-0136-1.

39. Fu J, Li K, Zhang W, Wan C, Zhang J, Jiang P, et al. Large-scale public data reuse to model immunotherapy response and resistance. Genome Med. 2020;12(1):21. https://doi.org/10.1186/s13073-020-0721-z.

40. Hoshida Y, Brunet JP, Tamayo P, Golub TR, Mesirov JP. Subclass mapping: identifying common subtypes in independent disease data sets. PLoS One. 2007;2(11):e1195. https://doi.org/10.1371/journal.pone.0001195.

41. Geeleher P, Cox N, Huang RS. pRRophetic: an R package for prediction of clinical chemotherapeutic response from tumor gene expression levels. PLoS One. 2014;9(9):e107468. https://doi.org/10.1371/journal.pone.0107468.

42. Tibshirani R. The lasso method for variable selection in the Cox model. Stat Med. 1997;16(4):385-95. https://doi.org/10.1002/(SICI) 1097-0258(19970228)1 6:4<385::AID-SIM380>3.0.CO;2-3.

43. Li H, Liu J, Chen J, Wang H, Yang L, Chen F, et al. A serum microRNA signature predicts trastuzumab benefit in HER2-positive metastatic breast cancer patients. Nat Commun. 2018;9(1):1614. https://doi.org/10.1038/s414 67-018-03537-W.

44. Wang Y-Q, Zhang Y, Jiang W, Chen Y-P, Xu S-Y, Liu N, et al. Development and validation of an immune checkpoint-based signature to predict 
prognosis in nasopharyngeal carcinoma using computational pathology analysis. J Immunother Cancer. 2019;7(1):298. https://doi.org/10.1186/s4042 5-019-0752-4.

45. Zhang $Y$, Zhang $L, X u Y$, Wu X, Zhou Y, Mo J. Immune-related long noncoding RNA signature for predicting survival and immune checkpoint blockade in hepatocellular carcinoma. J Cell Physiol. 2020;235(12):9304-16. https://doi.org/10.1002/jcp.29730.

46. Mao X, Qin X, Li L, Zhou J, Zhou M, Li X, et al. A 15-long non-coding RNA signature to improve prognosis prediction of cervical squamous cell carcinoma. Gynecol Oncol. 2018;149(1):181-7. https://doi.org/10.1016/j. ygyno.2017.12.011

47. Zhu X, Tian X, Yu C, Shen C, Yan T, Hong J, et al. A long non-coding RNA signature to improve prognosis prediction of gastric cancer. Mol Cancer. 2016;15(1):60. https://doi.org/10.1186/s12943-016-0544-0.

48. Cai J, Li B, Zhu Y, Fang X, Zhu M, Wang M, et al. Prognostic biomarker identification through integrating the gene signatures of hepatocellular carcinoma properties. EBioMedicine. 2017;19:18-30. https://doi.org/10.1016/j. ebiom.2017.04.014.

49. Fluss R, Faraggi D, Reiser B. Estimation of the Youden index and its associated cutoff point. Biom J. 2005;47(4):458-72. https://doi.org/10.1002/ bimj.200410135.

50. Cai $C$, Yang L, Tang $Y$, Wang $H, H e ~ Y$, Jiang $H$, et al. Prediction of overall survival in gastric Cancer using a nine-IncRNA. DNA Cell Biol. 2019;38(9): 1005-12. https://doi.org/10.1089/dna.2019.4832.

51. Zhang Y, Chen S-W, Liu L-L, Yang X, Cai S-H, Yun J-P. A model combining TNM stage and tumor size shows utility in predicting recurrence among patients with hepatocellular carcinoma after resection. Cancer Manag Res. 2018;10:3707-15. https://doi.org/10.2147/CMAR.S175303.

52. Kitagawa M, Kitagawa K, Kotake Y, Niida H, Ohhata T. Cell cycle regulation by long non-coding RNAs. Cell Mol Life Sci. 2013;70(24):4785-94. https://doi. org/10.1007/s00018-013-1423-0

53. Erdag G, Schaefer JT, Smolkin ME, Deacon DH, Shea SM, Dengel LT, et al. Immunotype and immunohistologic characteristics of tumor-infiltrating immune cells are associated with clinical outcome in metastatic melanoma. Cancer Res. 2012;72(5):1070-80. https://doi.org/10.1158/0008-5472.CA N-11-3218.

54. Galon J, Costes A, Sanchez-Cabo F, Kirilovsky A, Mlecnik B, Lagorce-Pages C, et al. Type, density, and location of immune cells within human colorectal tumors predict clinical outcome. Science. 2006;313(5795):1960-4. https://doi. org/10.1126/science.1129139.

55. Santoiemma PP, Powell DJ Jr. Tumor infiltrating lymphocytes in ovarian cancer. Cancer Biol Ther. 2015;16(6):807-20. https://doi.org/10.1080/1538404 7.2015.1040960

56. Shirabe K, Matsumata T, Maeda T, Sadanaga N, Kuwano H, Sugimachi K. A long-term surviving patient with hepatocellular carcinoma including lymphocytes infiltration--a clinicopathological study. Hepatogastroenterology. 1995;42(6):996-1001.

57. Wada Y, Nakashima O, Kutami R, Yamamoto O, Kojiro M. Clinicopathological study on hepatocellular carcinoma with lymphocytic infiltration. Hepatology. 1998;27(2):407-14. https://doi.org/10.1002/hep.510270214.

58. Shen $Y$, Peng $X$, Shen $C$. Identification and validation of immune-related IncRNA prognostic signature for breast cancer. Genomics. 2020;112(3):26406. https://doi.org/10.1016/j.ygeno.2020.02.015.

59. Shrestha R, Prithviraj P, Anaka M, Bridle KR, Crawford DHG, Dhungel B, et al. Monitoring immune checkpoint regulators as predictive biomarkers in hepatocellular carcinoma. Front Oncol. 2018;8:269. https://doi.org/10.3389/ fonc.2018.00269.

60. Roh W, Chen PL, Reuben A, Spencer CN, Prieto PA, Miller JP, et al. Integrated molecular analysis of tumor biopsies on sequential CTLA-4 and PD-1 blockade reveals markers of response and resistance. Sci Transl Med. 2017;9(379):eaah3560.

61. Hon CC, Ramilowski JA, Harshbarger J, Bertin N, Rackham OJ, Gough J, et al. An atlas of human long non-coding RNAs with accurate $5^{\prime}$ ends. Nature. 2017;543(7644):199-204. https://doi.org/10.1038/nature21374.

62. Ruan $X$, Li P, Chen Y, Shi Y, Pirooznia M, Seifuddin F, et al. In vivo functional analysis of non-conserved human IncRNAs associated with cardiometabolic traits. Nat Commun. 2020;11(1):45. https://doi.org/10.1038/s41467-019-13 688-z.

63. Batista PJ, Chang HY. Long noncoding RNAs: cellular address codes in development and disease. Cell. 2013;152(6):1298-307. https://doi.org/10.101 6/j.cell.2013.02.012
64. Chi Y, Wang D, Wang J, Yu W, Yang J. Long non-coding RNA in the pathogenesis of cancers. Cells. 2019;8(9):1015. https://doi.org/10.3390/ cells8091015.

65. Imamura H, Matsuyama Y, Tanaka E, Ohkubo T, Hasegawa K, Miyagawa S, et al. Risk factors contributing to early and late phase intrahepatic recurrence of hepatocellular carcinoma after hepatectomy. J Hepatol. 2003; 38(2):200-7. https://doi.org/10.1016/S0168-8278(02)00360-4.

66. Shah SA, Greig PD, Gallinger S, Cattral MS, Dixon E, Kim RD, et al. Factors associated with early recurrence after resection for hepatocellular carcinoma and outcomes. J Am Coll Surg. 2006;202(2):275-83. https://doi.org/10.1016/j. jamcollsurg.2005.10.005.

67. Jung SM, Kim JM, Choi GS, Kwon CHD, Yi NJ, Lee KW, et al. Characteristics of early recurrence after curative liver resection for solitary hepatocellular carcinoma. J Gastrointest Surg. 2019;23(2):304-11. https://doi.org/10.1007/ s11605-018-3927-2.

68. Ward M, McEwan C, Mills JD, Janitz M. Conservation and tissue-specific transcription patterns of long noncoding RNAs. J Hum Transcr. 2015;1(1):29. https://doi.org/10.3109/23324015.2015.1077591.

69. Evangelou K, Havaki S, Kotsinas A. E2F transcription factors and digestive system malignancies: how much do we know? World J Gastroenterol. 2014; 20(29):10212-6. https://doi.org/10.3748/wjg.v20.i29.10212.

70. Stine ZE, Walton ZE, Altman BJ, Hsieh AL, Dang CV. MYC, metabolism, and Cancer. Cancer Discov. 2015;5(10):1024-39. https://doi.org/10.1158/2159-82 90.CD-15-0507.

71. Lobrich M, Jeggo PA. The impact of a negligent G2/M checkpoint on genomic instability and cancer induction. Nat Rev Cancer. 2007;7(11):861-9. https://doi.org/10.1038/nrc2248.

72. Gavande NS, VanderVere-Carozza PS, Hinshaw HD, Jalal SI, Sears CR, Pawelczak KS, et al. DNA repair targeted therapy: the past or future of cancer treatment? Pharmacol Ther. 2016;160:65-83. https://doi.org/10.1016/j. pharmthera.2016.02.003.

73. Pages F, Galon J, Dieu-Nosjean MC, Tartour E, Sautes-Fridman C, Fridman WH. Immune infiltration in human tumors: a prognostic factor that should not be ignored. Oncogene. 2010;29(8):1093-102. https://doi.org/10.1038/ onc.2009.416.

74. Garnelo M, Tan A, Her Z, Yeong J, Lim CJ, Chen J, et al. Interaction between tumour-infiltrating B cells and T cells controls the progression of hepatocellular carcinoma. Gut. 2017;66(2):342-51. https://doi.org/10.1136/ gutjnl-2015-310814.

75. Chew V, Chen J, Lee D, Loh E, Lee J, Lim KH, et al. Chemokine-driven lymphocyte infiltration: an early intratumoural event determining long-term survival in resectable hepatocellular carcinoma. Gut. 2012;61(3):427-38. https://doi.org/10.1136/gutjnl-2011-300509.

76. Cancer Genome Atlas Research Network, Electronic address wbe, Cancer Genome Atlas Research N. Comprehensive and integrative genomic characterization of hepatocellular carcinoma. Cell. 2017;169(7):1327-41 e23. https://doi.org/10.1016/j.cell.2017.05.046

\section{Publisher's Note}

Springer Nature remains neutral with regard to jurisdictional claims in published maps and institutional affiliations.

Ready to submit your research? Choose BMC and benefit from:

- fast, convenient online submission

- thorough peer review by experienced researchers in your field

- rapid publication on acceptance

- support for research data, including large and complex data types

- gold Open Access which fosters wider collaboration and increased citations

- maximum visibility for your research: over $100 \mathrm{M}$ website views per year

At $\mathrm{BMC}$, research is always in progress.

Learn more biomedcentral.com/submission 